\title{
Recent progress in simulations of the paramagnetic state of magnetic materials
}

Igor Abrikosov, A. V. Ponomareva, Peter Steneteg, S. A. Barannikova and Björn Alling

\author{
Linköping University Post Print
}

Tweet

N.B.: When citing this work, cite the original article.

Original Publication:

Igor Abrikosov, A. V. Ponomareva, Peter Steneteg, S. A. Barannikova and Björn Alling, Recent progress in simulations of the paramagnetic state of magnetic materials, 2016, Current opinion in solid state \&amp; materials science, (20), 2, 85-106.

http://dx.doi.org/10.1016/j.cossms.2015.07.003

Copyright: Elsevier

\section{http://www.elsevier.com/}

Postprint available at: Linköping University Electronic Press

http://urn.kb.se/resolve?urn=urn:nbn:se:liu:diva-127416

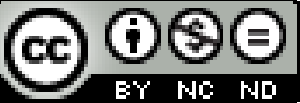


Recent progress in simulations of the paramagnetic state of magnetic materials I. A. Abrikosov, ${ }^{1,2,{ }^{*}}$ A. V. Ponomareva, ${ }^{2}$ P. Steneteg, ${ }^{3}$ S. A. Barannikova, ${ }^{2,4,5}$ B. Alling ${ }^{1}$

${ }^{1}$ Department of Physics, Chemistry and Biology (IFM), Linköping University, 58381 Linköping, Sweden

${ }^{2}$ Materials Modeling and Development Laboratory, National University of Science and Technology 'MISIS’, 119049 Moscow, Russia,

${ }^{3}$ Department of Science and Technology (ITN), Linköping University, 60174 Norrköping, Sweden

${ }^{4}$ National Research Tomsk State University, 634050 Tomsk, Russia.

${ }^{5}$ Institute of Strength Physics and Materials Science, SB RAS, 634055Tomsk, Russia.

*Corresponding author, e-mail address: igor.abrikosov@ifm.liu.se

\begin{abstract}
We review recent developments in the field of first-principles simulations of magnetic materials above the magnetic order-disorder transition temperature, focusing mainly on $3 d$-transition metals, their alloys and compounds. We review theoretical tools, which allow for a description of a system with local moments, which survive, but become disordered in the paramagnetic state, focusing on their advantages and limitations. We discuss applications of these theories for calculations of thermodynamic and mechanical properties of paramagnetic materials. The presented examples include, among others, simulations of phase stability of Fe, Fe-Cr and Fe-Mn alloys, formation energies of vacancies, substitutional and interstitial impurities, as well as their interactions in Fe, calculations of equations of state and elastic moduli for $3 d$-transition metal alloys and compounds, like CrN and steels. The examples underline the need for a proper treatment of magnetic disorder in these systems.
\end{abstract}

Keywords: Paramagnetic state, magnetic materials, first-principles simulations

\title{
1. Introduction: a description of the paramagnetic state of magnetic materials.
}

Materials play very important role in the history of humankind. In the 1900-s the advent of electricity, aviation, nuclear power, and information technology was governed by the explosion of materials discoveries. Plastic and silicon, superconductors and biomaterials, photonic materials and ceramic composites became available. Moreover, accelerating technological development greatly increased demands for the materials design. So far, the prevailing search methods were based on trial-and-error development. However, the time it takes to discover 
advanced materials and to prove their usefulness to a commercial market is far too long. Thus, there is a need to reduce it significantly, from 10-20 years at present to 5-10 years or less [1]. Theoretical understanding of fundamental properties of materials and a possibility to carry out advanced computer simulations of materials properties should be considered as a key in achieving this goal.

A challenging problem for the condensed matter theory in this respect is to bring simulations as close as possible to conditions at which materials operate when used as tools and devices. Indeed, the physical and mechanical properties of materials depend on the chemical content, on the internal structure, which is formed during their manufacturing and service, as well as on temperature, stresses, and other external parameters. In the case of a magnetic material the situation becomes even more complex, turning fundamental study of magnetism into a subject of great scientific and practical interest, and leading to enormous amount of experimental and theoretical investigations in this field [2].

Great progress has been achieved in understanding of magnetically ordered materials. Consideration of relatively trivial types of collinear magnetic order, ferromagnetic, with all magnetic moment pointing in the same direction, or simple antiferromagnetic, say with magnetic moments in neighboring planes of the crystal pointing antiparallel to each other, is nowadays extended to significantly more rich spin textures, e.g. helical and skyrmion structures [3]. First principles calculations in the framework of Density Functional Theory (DFT) [4] have been recognized in the field as an extremely useful research tool. With a development of efficient computational methods [5] and increasing power of modern computers calculations of key magnetic characteristics, like the local magnetic moments or magnetic exchange interactions, have transformed into a routine task, and theoretical treatments of noncollinear spin configurations [6,7], magnetic phase transitions [8], and spin dynamics [9, 10, 11, 12,13] have become possible.

An interplay between magnetic and chemical effects in magnetic materials was recognized several decades ago $[14,15]$. Unfortunately, the effects of finite temperature magnetic excitations on their structural and elastic properties attracted much less attention. Often these effects were assumed small, the second-order effects, which should not be taken into account in simulations of phase stability and mechanical behavior. Moreover, paramagnetic phases of magnetic materials were modeled as non-magnetic in many works, and researchers did not distinguish between the two terms. Such misinterpretation could lead to erroneous conclusions [16]. Indeed, in most cases local magnetic moments survive above the magnetic transition temperature, seriously modifying the picture obtained in theoretical simulations [17]. The proper treatment of magnetic disorder is essential for the predictive description of materials properties $[18,19]$, especially just below or above the Curie temperature [20].

Early attempts to understand the behavior of magnetic materials were dominated by two seemingly orthogonal pictures, the local magnetic moments model and the itinerant (band electrons) model. The former could be traced to the work of Heisenberg [21], and assumed that electrons were localized on atoms producing a local spin moment. The inter-atomic exchange interactions determined the magnetic order, and transverse spin fluctuations were excited at finite temperature, eventually leading to a disorder of the localized moments above the magnetic 
transition temperature. The itinerant model was based on the band theory of electrons, and was best represented by the Stoner description [22]. The competition between the kinetic energy of the itinerant electrons and the exchange interaction between them could give rise an imbalance in the numbers of spin up and spin down electrons, stabilizing a magnetic order, e.g.

ferromagnetism. The longitudinal components of the local spin fluctuation lead to the variation of the magnetic moment with temperature and dominated the thermodynamic properties of magnetic materials.

Experimental data for the Curie-Weiss constant and the saturation magnetization of a wide variety of ferromagnetic substances were used by Rhodes and Wohlfarth to calculate values for the numbers of magnetic carriers from the former and from the latter, $q_{C}$ and $q_{S}$, respectively [23]. Plotting their ratio as a function of the Curie temperatures of corresponding materials, Rhodes and Wohlfarth revealed two branches, one with $q_{C} / q_{S} \sim 1$, and the other with $q_{C} / q_{S}>1$. The former corresponded to substances, such as $\mathrm{Gd}$ and MnSb, which could be described with a purely localized model of ferromagnetism. The second branch corresponded to substances, such as nickel and its alloys, dilute alloys of palladium and the compounds $\mathrm{Sc}_{3} \mathrm{In}$ and $\mathrm{ZrZn}_{2}$, which Rhodes and Wohlfarth classified according to collective electron model of ferromagnetism.

Probably, purely localized or the purely itinerant moment models could describe some materials. However, in general any of them alone should not be sufficient. Nowadays we understand that itinerant electrons determine the magnetic properties of transition metals and their alloys.

Electronic structure calculations within DFT, which can be viewed as a modern extension of the Stoner-type description of magnetism, are capable to reproduce ground state magnetic properties of $3 d$ transition metals and their alloys with very high accuracy, and to explain them [24]. However, applications of the Stoner picture fail for the description of magnetism at finite temperature. It greatly overestimates the Curie temperatures for ferromagnetic metals $T_{c}$, by factor of five, and there are no moments and no Curie-Weiss law above $T_{c}$ [25].

Very important step in the development of modern understanding of transition metal magnetism can be traced back to works of Moriya, who resolved the controversy between the itinerant and localized models into a more general problem of spin density fluctuations [26]. His interpolation theory was based on the functional integral formalism and the average amplitude of the local spin fluctuation was taken as one of the most important physical variables. Moriya has developed a method of taking into account the nonlocal nature of spin fluctuations so that the local moment limit and the weakly ferro- and antiferromagnetic limit were properly interpolated.

Moriya's works inspired the development of several first-principles approaches for the description of paramagnetic materials that we review in this paper. We restrict ourselves with a discussion of $3 d$-series transition metals, their alloys and compounds, to limit nearly endless field to a manageable amount of information. However, the approaches and concepts that we discuss have sufficient generality, and should be applicable to a broad set of substances. Our starting point can be described as follows. Itinerant electrons determine the magnetism of $3 d$-transition metals. However, they are relatively strongly bounded to their sites. Figure 1 illustrates that the magnetization density in these systems is well localized. Consequently, each atom could be associated with a local moment parallel to the net magnetization density at the site. These local moments behave in a Heisenberg-like manner, that is like localized moments, and become 


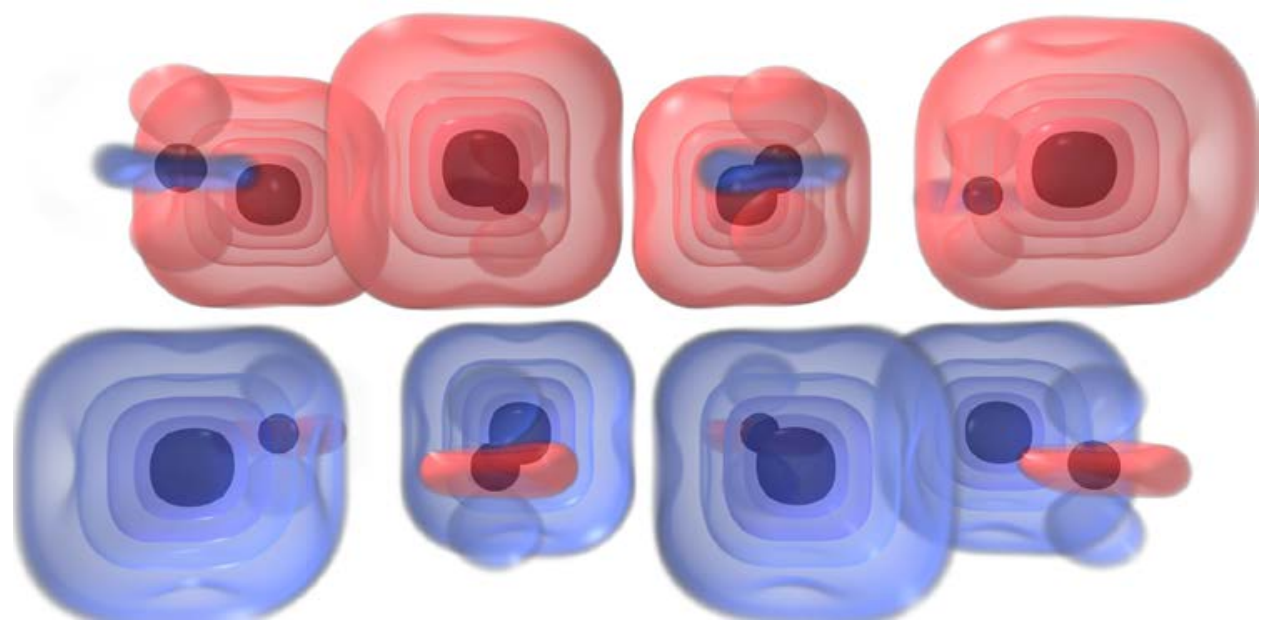

Figure 1. Magnetization density calculated for orthorhombic antiferromagnetic phase of $\mathrm{CrN}$ simulated by $2 \times 1 \times 1$ unit cells. The density is shown by iso-surfaces at $0.06,0.57,1.14$, and 1.71 electrons / $\AA^{3}$. Red (blue) colors correspond to a surplus of majority (minority) spin electrons. It is seen that the magnetization density is well localized at $\mathrm{Cr}$ atoms (large bulbs) though some induced spin polarization at $\mathrm{N}$ atoms is also seen. Details of calculations are the same as in Ref. [27].

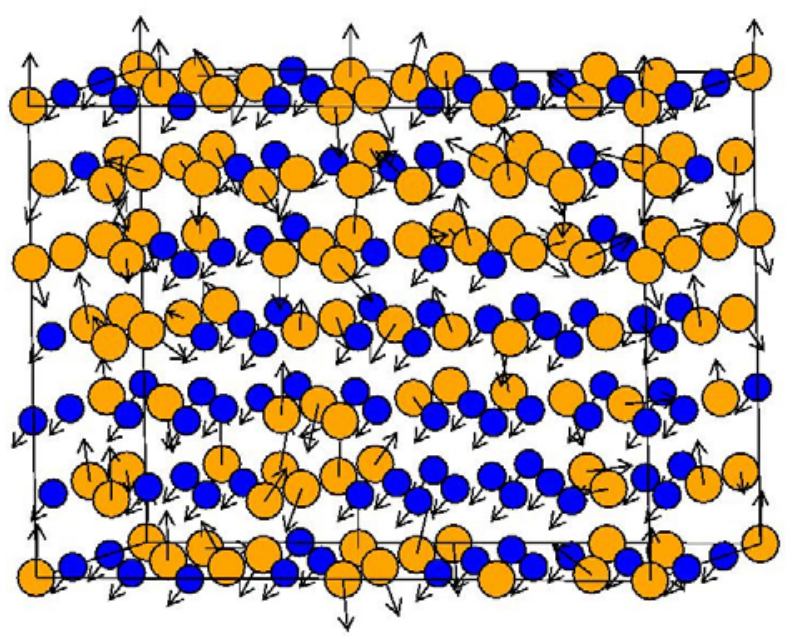

Figure 2. The model of the paramagnetic state considered in this work consists of non-collinear and fluctuating local moments, which become disordered above the magnetic order-disorder transition temperature, the Cure $T_{C}$ or Neel $T_{N}$ temperature for ferromagnets and antiferromagnets, respectively. 
disordered above the magnetic order-disorder transition temperature, the Cure $T_{C}$ or Neel $T_{N}$ temperature for ferromagnets and antiferromagnets, respectively, as illustrated in Fig. 2. Of course, in weak itinerant magnets with $q_{C} / q_{S}>>1$ like $\mathrm{ZrZn}_{2}$, this picture does not hold. Here the magnetization arises because of specific narrow peaks in the electronic density of states at the Fermi level, fulfilling the Stoner criteria. Under such circumstances, the concept of the moments rotating nearly rigidly probably breaks down and the excitations can be more from electron-hole pairs than from moment rotations [10]. We will not consider weakly itinerant magnets in this review.

At the same time, magnitudes of the local moments do not have to be constant even for chemically equivalent atoms. They depend on the local chemical environments of the respective atoms, e.g. due to disorder in alloys or the proximity to defects, on thermally induced atomic displacements, as well as on local magnetic environments of the atoms. Moreover, formed by itinerant electrons magnetic moments fluctuate in space and time due to the presence of longitudinal spin fluctuation. These fluctuations are closely linked to the itinerant nature of electron magnetism, where electron-electron exchange interaction is responsible for the formation of local atomic magnetic moments [13]. The longitudinal fluctuations have a significant effect on the high-temperature properties of magnetic materials, influencing the description of their finitetemperature thermodynamics [28]. Longitudinal spin fluctuations can be incorporated into the local moment picture, either self-consistently, at the level of DFT calculations, by replacing the Weiss field in the calculation with a generalized Onsager cavity field in the framework of the disordered local moment picture [29], or at a level of model Hamiltonians [30-34].

An approach described above has been widely used for studies of finite temperature magnetism, but mainly in the context of simulations of magnetic properties, like $T_{C}$ and $T_{N}$. On the other hand, first-principles simulations of phase stability, thermodynamic and elastic properties of magnetic materials above these temperatures is a relatively young field, despite the fact that Zener underlined the importance of magnetic excitations for the allotropic phase transitions in $\mathrm{Fe}$ almost 60 years ago [35]. Körmann et al. have given a comprehensive overview of state-of-theart computational techniques to thermodynamically model magnetic and chemical order-disorder transitions [28]. Reviewing recent progress in this field with a focus on first-principles description of paramagnetic materials based on $3 d$-series transition metals is the main task of this work. 


\section{Theoretical models for description of paramagnetic state of magnetic materials: general considerations.}

To understand the complexity of the problem, let us consider a magnetic material above its Curie or Neel temperature that is in the paramagnetic phase. To describe its thermodynamic properties one must determine the Gibbs free energy:

$G_{P M}=G_{e l}+G_{v i b}+G_{m a g n}$,

which contains electronic $G_{e l}$, vibrational $G_{v i b}$, and magnetic $G_{m a g n}$ contributions. State-of-the art approach to this type of tasks would be to employ the adiabatic decoupling between the three degrees of freedom, motivating it by the fact that their excitations usually have very different time scales [36]. The separation of the electronic, the fastest, and atomic vibration degrees of freedom are well justified within the Born-Oppenheimer approximation, assuming that lighter electrons adjust adiabatically to the motion of much heavier nuclei, remaining at any time in their instantaneous ground state. Indeed, as discussed in [25] the characteristic time for $d$-metals as given by intersite hopping is $\sim 10^{-15} \mathrm{~s}$, while for the lattice vibrations the characteristic time is proportional to the inverse of the Debye frequency, which is of the order of $\sim 10^{-12} \mathrm{~s}$. Excitations associated with magnetic degrees of freedom are commonly related to the to the inverse spinwave frequency, which is $\sim 10^{-13} \mathrm{~s}$, and therefore they are assumed to be order of magnitude faster than vibrational degrees of freedom, and several orders of magnitude slower than the electronic ones.

On the other hand, in the paramagnetic state the magnetic excitations are qualitatively different from those at the low temperature. In contrast to the spin wave excitations, other transverse as well as longitudinal excitations of the magnetic moments, like the spin flips, dominate. The relevant time scale can therefore be better estimated from the spin decoherence time $t_{d c}$ rather than from the inverse spin-wave frequency. For body-centered cubic ( $b c c)$ Fe above $T_{C} t_{d c}$ is of the order of 20-50 fs [11]. Thus, the magnetic degrees of freedom in the paramagnetic state should be considered as much faster in comparison to those in magnetically ordered materials, of the order of $10^{-14}-10^{-15} \mathrm{~s}$. Moreover, it is important to realize that the paramagnetic state is the high-temperature state of a magnetic material. Thus, a consideration of atomic motions is essential. The time scale given by the Debye frequency is therefore getting less relevant, and one should consider the atomic motions on the time scales familiar from molecular dynamics simulations. Here the typical time step for a proper description of the (Born-Oppenheimer) dynamics is $\sim 10^{-15} \mathrm{~s}$. Finally, the potential energy surface can be influenced by magnetic and vibrational excitations. In summary, an argument for the decoupling of electronic, magnetic and vibrational contributions to the free energy, well justified for magnetically ordered materials at low temperatures becomes questionable for the theoretical description of their paramagnetic state. 
All the terms in Eq. (1), $G_{e l}, G_{v i b}$, and $G_{m a g n}$ are coupled to each other and in principle should be treated simultaneously in first-principles calculations of paramagnetic materials.

To complicate the picture further, it is important to underline that the physics of strongly correlated electron systems recognizes the description of the finite-temperature itinerant electron magnets and the existence of local magnetic moments above $T_{C}$ or $T_{N}$ as one of the central problems [37]. For a system with itinerant electrons local magnetic moments become temperature dependent. Besides the thermal fluctuations which disorder the moments, a variety of competing many-body effects should be considered, such as Kondo screening and the induction of local magnetic moment by temperature. These effects go beyond the state-of-the-art local (local spin density approximation, LSDA) or semi-local (generalized gradient approximation, GGA) implementations of DFT, and require applications of advanced, but time-consuming techniques, e.g. based on the dynamical mean-field theory (DMFT) [38].

Thus, the problem of the description of the paramagnetic state of magnetic materials appears to be challenging for the first-principles theory. However, the great practical importance of e.g. steels has motivated intense research in the field, and several research groups have dealt with it, from different starting points. In particular, DMFT calculations have been carried out for several systems, despite their complexity. On the other hand, it has been realized that some of the manybody effects can be taken into account, in an approximate way, in the framework of more traditional density functional techniques. Below we discuss these theoretical developments.

\section{Dynamical Mean-Field Theory.}

Perhaps the most consistent way to describe the paramagnetic state of a magnetic material with account of correlation effects is given at present by a combination of DFT with model treatments of many-body effects, like the Hubbard model, e.g. within the dynamical mean-field theory. The DMFT [39] maps the Hubbard model, which in principle is a lattice model, onto quantum impurity model subject to a self-consistent condition in such a way that the many-body problem for the crystal splits into a one-body impurity problem for the crystal and a many-body problem for an effective atom. The relevant degrees of freedom at a single site are the quantum states of the atom, while the rest of the crystal is described as a reservoir of non-interacting electrons that can be emitted or absorbed in the atom [40]. The effect of the environment on the site is to allow the atom to make transitions between different configurations. The quantum nature of the problem requires a hybridization function that plays the role of a mean field and describes the ability of electrons to hop in and out of a given atomic site, ensuring a self-consistency condition to the quantum impurity problem. In the case of weak hybridization the electrons will behave as localized, while in the opposite case they will behave as itinerant. The field is determined selfconsistently: one uses the solution of the quantum many-body impurity problem to build self- 
energy for the lattice Green's function that in turn gives a new approximation for the dynamical mean-field. A combination of DFT and DMFT is realized in the following way: the electronic structure calculation results obtained by DFT methods are used to calculate parameters for a general DMFT Hamiltonian and then the problem defined by this Hamiltonian is solved within the DMFT [41]. More detailed introductory information on the DMFT can be found in [40], fundamental basics of DMFT have been reviewed by Kotliar et al.[38], while a review on recent investigation of real materials with strong electronic correlations by the LDA+DMFT method has been presented by Anisimov and Lukoyanov[41].

Consider the problem of the paramagnetic state of magnetic materials. Solving the quantum impurity problem within DMFT, one describes the spin, orbital, energy, and temperature dependent interactions of a particular magnetic atom with the rest of the crystal. The uniform spin susceptibility in the paramagnetic state $\chi_{q=0}=d M / d H$ can be extracted from Quantum MonteCarlo (QMC) simulations [37] used for the solution of DMFT quantum impurity problem by measuring the induced magnetic moment in a small external magnetic field. It includes the polarization of the impurity Weiss field by the external field. For a Fermi liquid one expects

temperature independent Pauli behavior of $\chi_{q=0}$. On the other hand, if the local moments survive above the magnetic transition temperature, the uniform spin susceptibility follows the CurieWeiss law:

$$
\chi_{q=0}=\frac{\mu_{e f f}^{2}}{3\left(T-T_{C}\right)} \quad,
$$

which allows one to extract high-temperature magnetic moments $\mu_{\text {eff }}$ and the magnetic transition temperature $T_{C}$ from calculations. Of course, the latter should be overestimated due to the meanfield approximation, underlying the DMFT.

Another important quantity that one can compute using the LDA+DMFT approach for a paramagnetic system is the dynamic local magnetic susceptibility [42]:

$$
\chi_{\text {loc }}(i \omega)=\mu_{B}^{2} \int_{0}^{\beta} d \tau\left\langle S_{j}^{z}(0) S_{j}^{z}(\tau)\right) e^{i \omega \tau},
$$

where $\mu_{B}$ is the Bohr magneton, $S_{j}^{z}$ is the $z$-component of single-site spin operator $\boldsymbol{S}$ at site $j$, and $\beta=T^{-1}$. Taking analytical continuation of $\chi_{\text {loc }}(i \omega)$ to the real frequency axis $\omega$, one can try to fit $\operatorname{Re} \chi_{\text {loc }}$ by the simple Lorentzian form, denoting half-width of the peak at a half-height as $\delta . \delta$ describes the inverse lifetime of local excitations, and the large is it the smaller is lifetime of the local moments in the paramagnetic state. The static susceptibility in the local-moment regime is given by $\chi_{\text {loc }}(0)$. In contrast to the uniform spin susceptibility, the dynamic local magnetic 
susceptibility cannot be measured in experiments, but its temperature dependence characterizes the degree of correlations in the system. $\chi_{\text {loc }}$ should be nearly temperature independent in weakly correlated systems, while in strongly correlated systems one expects the Curie-Weiss behavior at high temperatures [37]:

$\chi_{\text {loc }}=\frac{\mu_{l o c}^{2}}{(3 T+c o n s t)}$

For the system with well-defined local moments the DMFT is expected to yield $\delta \sim T$ and $\mu_{\text {loc }}$ should be temperature independent, while in the more itinerant system one should see significant temperature dependence of $\mu_{\text {loc }}$.

In original applications of the LDA+DMFT the procedure was carried out without charge selfconsistency. However, state-of-the-art applications of the method include the charge selfconsistency, making it possible to calculate the total energy and to access thermodynamic [43] and vibrational [44] properties of paramagnetic materials in addition to the magnetic properties. At the same time, it is important to understand that the DMFT itself contains several approximations, which should be well understood in its practical applications. Being the meanfield theory, it neglects intersite magnetic exchange, and it should in general overestimate magnetic transition temperature. Magnetic short-range order present in any system not too far above the magnetic transition temperature cannot be captured as well. Note that a comparison of the values of the local and the $q=0$ susceptibilities could give a crude measure of the degree of the short range order [37]. Methods to add nonlocal corrections to the DMFT have been developed, for example the dynamical cluster approximation $[45,46]$, the cellular dynamical mean-field theory [47], and the dual fermion approach [48]. However, their application in simulations relevant for the materials design are still illusive due to very high computational costs.

Another big problem for practical applications of the LDA+DMFT scheme is the existence of two parameters of the model, the Coulomb $U$ and exchange $J$ interaction parameters. Often these parameters are varied to achieve the best agreement between experimental data and calculations for some known and well-characterized property. Within such approach, the model parameters are considered as adjustable parameters. In principle, one can estimate the value for the Coulomb interaction parameter $U$ from the energy separation of the spectral peaks interpreted as Hubbard bands [41]. Moreover, there are computational schemes proposed to calculate $U$ and $J$ from first principles, e.g. employing the constrained random-phase-approximation (cRPA) method [49,50]. However, their use is still quite rare. Unfortunately, the LDA+DMFT calculations are still time consuming, and the method is most often applied to materials with few atoms in the unit cell and with relatively high degree of symmetry. Moreover, until recently forces between atoms were 
calculated from the numerical derivation of the total energies, ruling out efficient consideration of lattice dynamics. Fortunately, Leonov et al. [51] has just proposed a novel computational approach which makes it possible to evaluate interatomic forces within DMFT approach. Still, there is a need for theoretical methods that treat many-electron effects at more approximate level.

\section{Spin dynamics}

The key point for a theory that aims at simplifying the treatment of the many-body effects in paramagnetic materials is the equivalence between a many-body interacting system with Coulomb onsite interactions and a one-electron system in fluctuating charge and spin fields. This equivalence is a base of spin-fluctuation theories of itinerant-electron magnetism [26]. In a complete theory, the charge and spin fields are dynamically fluctuating in both space and time. One could try to capture these fluctuations with DFT calculations for a system which magnetic state is excited, and where the arrangement of local moments differs from that in the magnetically ordered ground state and fluctuates with time. Perhaps, ab initio spin dynamics gives the most consistent realization of this approach.

Antropov et al. [9] inspired the interest to this technique, and presented a detailed derivation of the equations about a year later [10]. Starting with basic DFT formalism and considering each electron moving in the average "charge" and "spin"' self-consistent fields $V(\boldsymbol{r})$ and $\boldsymbol{B}(\boldsymbol{r})$ of the electrons and ions, Antropov et al. derived the equation of motion (EOM) for spin density $\mathbf{m}(\mathbf{r}, t)$ :

$\frac{d \mathbf{m}(\mathbf{r}, t)}{t}=g_{s} \mathbf{m} \times \mathbf{B}+\frac{i}{2} \nabla(\Psi * \hat{\boldsymbol{\sigma}} \nabla \Psi-c . c)$

where $g_{s}$ is the gyromagnetic ratio, $\boldsymbol{\Psi}$ is the electronic spinor, $\hat{\boldsymbol{\sigma}}$ represents a vector of Pauli matrices, and c.c. stays for complex conjugate.

To make the solution of equation (5) possible in practice and to include temperature effects, Antropov et al. introduced the quasiclassical spin approximation [9,10]. They assumed that the magnetization density in the immediate vicinity of an atom has a uniform orientation, divided the space into spheres or polyhedra, and within each such region associated a unit vector $\mathbf{e}_{i}$ with the instantaneous magnetization direction. Associating such a region with one particular atom with the local moment $\mathbf{M}_{i}=\mu \mathbf{e}_{i}$, they arrived at the local moment picture, which we discussed in the introduction and illustrated in Fig. 2. The next simplification consisted in introducing the rigid spin approximation (RSA), assuming that the time evolution of the orientation is described by a simultaneous (or rigid) rotation of the magnetization density at each point inside the atomic 
sphere or polyhedra. Note that the amplitude of the magnetization density was allowed to change. With these approximations quasi-classical EOM of $a b$ initio spin dynamics take the form:

$$
\frac{d \mathbf{e}}{t}=-\frac{2}{\mu} \mathbf{e} \times \mathbf{I}
$$

where

$$
\mathbf{I}=-\mu \mathbf{B}=\partial E / \partial \mathbf{e}
$$

is the first variation of the total energy $E$ for a differential rotation of a local moment, and therefore in analogy with molecular dynamics can be viewed as a magnetic "force" acting on the spin. It can be calculated, for instance, using the magnetic force theorem within the multiple scattering formalism [52]. To include temperature effects, Eq. (6) can be modified, e.g. in the framework of a stochastic method based on Langevin-type dynamics [10]:

$$
\frac{d \mathbf{e}_{i}}{t}=-\frac{2}{\mu}\left[\mathbf{e}_{i} \times \mathbf{I}_{i}\right]+\mathbf{R}_{i}+\mathbf{f}_{i}
$$

In Eq. (8) $\mathbf{R}_{i}$ is a relaxation term and $\mathbf{f}_{i}$ is a random force at each site $i$ in the system.

Despite the simplifications mentioned above, an approach proposed by Antropov et al. remained computationally demanding. Simulations were performed, but for relatively simple systems, like pure Fe $[9,10]$ or FeNi alloys [6,7]. Further development of the idea was associated with bringing model Hamiltonians into play to calculate the magnetic "force" in Eq. (7). The simplest model can be based on the classical Heisenberg Hamiltonian $\mathbf{H}_{c H}$ :

$$
\mathbf{H}_{c H}=-\sum_{i, j \neq i} J_{i j} \mathbf{e}_{i} \mathbf{e}_{j}
$$

known to work well for transition metals and their alloys [52]. Of course, this means that one goes from quasi-classical to semi-classical description. Indeed, the exchange parameters $J_{i j}$ are calculated within DFT, thus linking first-principles theory to the classical spin-dynamics. Skubic et al. [12] presented an excellent overview of the semi-classical approach that greatly simplifies the problem. Moreover, they proposed to use more complex Hamiltonian as compared to Eq. (9), adding to the classical Heisenberg Hamiltonian terms corresponding to uniaxial magnetic anisotropy, dipolar interactions, and the Zeeman term that described the interaction of the magnetic system with an external magnetic field. 
One should note however, that while the semi-classical approximation was demonstrated as a useful tool to study spin dynamics in many systems [11,12], very limited number of simulation had a description of the paramagnetic state as the primary task [53]. Several issues have to be considered here, like the importance of longitudinal spin fluctuations, the dependence of exchange parameters on the global magnetic state of the system, deviations between the classical and quantum solution of the Heisenberg model, and the effect of lattice vibrations.

The lack of longitudinal fluctuations (LFs) was a fundamental drawback of the classical Langevin-type spin dynamics. Ma and Dudarev [13] proposed a scheme to include LFs in a semiclassical dynamics of evolution of interacting magnetic moments. Their method was based on the generalization of Langevin spin dynamics to a fully three-dimensional stochastic dynamics of moments. In this approach, both the longitudinal and rotational degrees of freedom of atomic spin vectors were treated on equal footing. This removed a fundamental limitation associated with the lack of longitudinal fluctuations in semi-classical spin dynamics equations, but retained the capacity of the method to simulate a very large system of interacting spins.

A key aspect in a development of semi-classical theories that incorporate the longitudinal spin fluctuations has been a generalization of the classical Heisenberg Hamiltonian (9). Several suggestions have been put forward, which could be classified in two groups. The first group was based on an idea of merging the Heisenberg Hamiltonian (9) with a Ginzburg-Landau like energy expansion into a Heisenberg-Landau Hamiltonian [13, 30, 31, 33, 34]:

$$
\mathbf{H}_{H L}=-\sum_{i, j \neq i} J_{i j} \mathbf{M}_{i} \mathbf{M}_{j}+\sum_{i} \sum_{n=0}^{n_{\max }} A_{i}^{(n)} M_{i}^{2 n},
$$

where $A_{i}^{(n)}$ were the parameters of the Ginzburg-Landau like energy expansion. The second suggestion by Ruban et al. [32] introduced the longitudinal spin-fluctuation Hamiltonian $\mathbf{H}_{\text {LSF }}$ by making the parameters of the classical Heisenberg-type Hamiltonian dependent on the magnitudes of local moments:

$$
\mathbf{H}_{L S F}=\sum_{i} J^{(0)}(\bar{M})+\sum_{i} J^{(1)}\left(\bar{M}, M_{i}\right)-\sum_{i, j \neq i} J_{i j}\left(\bar{M}, M_{i}, M_{j}\right) \mathbf{M}_{i} \mathbf{M}_{j} \quad,
$$

In both approaches, the parameters of the Hamiltonians should be fitted to constrained DFT calculations.

Ruban et al. [32] underlined another important aspect of the use of model Hamiltonians, the dependence of the model parameters on the global magnetic state. In particular, they calculated 
the parameters of Eq. (11) for the magnetically disordered state simulated with the Disordered Local Moment (DLM) model (see Sec. 5 below). Thus, the parameters of the model Hamiltonian in Eq. (11) have the following physical meaning. $J^{(0)}(\bar{M})$ corresponds to the energy of the magnetically disordered state, which represents a system with randomly oriented spins with a fixed value of the magnetic moment $\bar{M} . J^{(1)}\left(\bar{M}, M_{i}\right)$ is the energy required to change the value of the spin from the corresponding average value $\bar{M}$ to the value $M_{i}$. The pair exchange interaction parameter $J_{i j}$ describes the magnetic interaction between atoms in positions $i$ and $j$ in the system where all other local moments were completely disordered. Ruban derived an expression for calculation of $J_{i j}$ in the paramagnetic state [54] using the multiple scattering theory, which differed from that of Ref. [52], where it was derived for a ferromagnetic ground state. As a matter of fact, for many itinerant systems there were significant differences in the results, calculated with the two expressions [54,55]. The problem is well recognized by now, and several groups propose their own schemes for taking into account the dependence of the model Hamiltonians parameters on the global magnetic state for the itinerant systems $[56,57,58]$.

Another interesting question is associated with possible deviations between the classical and quantum solution of the Heisenberg model. This problem has been studied in Ref.[59]. Based on a comparison between numerically exact results obtained from spin QMC simulations and classical simulations for different model systems and various thermodynamic properties, Körmann et al. have concluded that below $T_{C}$ the classical treatment could deviate significantly compared to the quantum treatment. On the other hand, for high temperatures well above $T_{C}$ as well as for increasing spin quantum numbers quantities such as the internal energy and the specific-heat capacity have approached each other as expected.

\section{Models of the paramagnetic state based on static DFT calculations at zero temperature.}

\subsection{Disordered Local Moment model in the framework of the coherent potential approximation.}

Disordered Local Moment model was introduced by Hubbard [60] and Hasegawa [61]. Gyorffy et al. [25] gave the modern derivation of the DLM and combined it with the LSDA-DFT. Within this model one assumes that the system consisting of all the electrons, although ergodic, does not cover its phase space uniformly in time. Rather, it gets stuck for long times, which we here denote as the spin-flip time $t_{S F}$, near points characterised by a finite moment at every site pointing in more or less random directions and then moves rapidly to another similar point. Gyorffy et al. supposed that the motion of temporarily broken ergodicity is mainly characterised by changes in the orientational configuration of the moments. Furthermore, Gyorffy et al. demonstrated [25] that neglecting the spin-orbit interaction and assuming the complete disorder between the local 


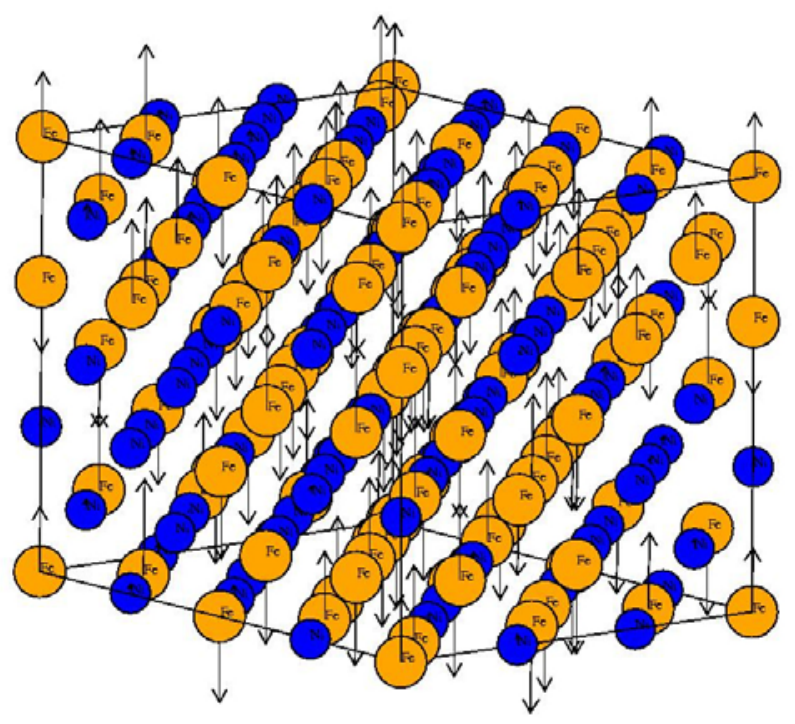

Figure 3. Schematic representation of the Disordered Local Moment model. The paramagnetic state consisting of fully disordered non-collinear and fluctuating magnetic moments (Fig. 2) is approximated by fully disordered, collinear and static picture.

moments the noncollinearity of the local moments (Fig. 2) could be ignored. Note that the model of complete disorder should work well at temperatures $T \gg J_{i j}^{\max }$, where $J_{i j}^{\max }$ is the strongest interaction of the classical Heisenberg Hamiltonian (9) for the corresponding system. Thus, within the DLM picture, the local magnetic moments exist in the paramagnetic state above the magnetic transition temperature, but the are fully disordered and collinear (Fig. 3). With this simplification, the magnetically disordered state can be described as a pseudo-alloy of equal amounts of atoms with spin up and spin down orientations of their local moments, and its electronic structure and the total energy can be calculated within the conventional alloy theory using the coherent potential approximation (CPA) [25].

A great advantage of the DLM picture consists of the possibility to formulate a consistent electronic-structure based thermodynamic theory, that accounts for the interplay between the configurational and magnetic degrees of freedom. In particular, Gyorffy and Stocks [62], and then Staunton et al.[63] derived a general formalism to describe the atomic short range order in the compositionally disordered phases, the so-called $S^{(2)}$ method. $S^{(2)}$ is defined as the second derivative with respect to concentration of the grand potential of the disordered alloy, and its physical meaning is related to the effective energy of the interchange of atoms in the alloy, the so-called effective cluster interactions [36]. If calculated in the reciprocal space, $S^{(2)}$ is directly 
related to the lattice Fourier transform of the short-range order parameter. In Ref. [54] Ruban et al. combined the corresponding real space appraoch, the generalized perturbation method, with the DLM model. These approaches allowed for the description of the configurational thermodynmaics in many alloys (for the recent review, see Ref.[36]).

Status of the DLM approach in the many-body lattice models like the Hubbard or s-f exchange "Kondo lattice" model was discussed, e.g. in Ref. [64]. It was argued that though in a complete theory the charge and spin fields were dynamically fluctuating both in space and time, a "static" DLM approximation, where one neglected the dynamics of the fluctuations might capture an important part of the correlations. Indeed, DLM combined with the CPA became equivalent to the "Hubbard III" approximation for the original many-body problem, which jusified the use of the DLM for systems with dominating transverse magnetic fluctuations and explained its success in many applications, ranging from basic simulations of magnetic phase transitions [25] to the design of new steels[65] and hard coatings [66,67]. Moreover, the DLM approach can be combined with techniques, that go beyond the conventional LSDA or GGA implementations of the DFT in terms of the tretment of many-body effects. In particular, it can be easily combined with the so-called LDA+U method [41,68,69], the static mean-field approximation (unrestricted Hartree-Fock) to the many-body problem. LDA+U method was found to be very successful when applied to systems with long-range spin and orbital order, but it was considered unsuitable for the desription of the paramagnetic state of correlated magnetic materials [70,71]. Alling et al. $[17,72]$ demonstrated that a combination of LDA+U with the DLM picture allowed them to lift off this restriction. We should discuss this problem in more details in Sec. 7.4.

On the other hand, DLM-CPA has other limitations, besides the approximate treatment of the many body effects. The codes that implement this approach are most often based on multiplescattering formalism and involve other approximations, e.g., the spherical approximation for the one-electron potential. This limits a possibility to treating materials with complex underlying crystal lattices and alloys with significant size mismatch between the alloy components, systems with point defects, like vacancies and interstitial impurities, or extended defects, like interfaces, grain boundaries and stacking faults. If local lattice relaxations are large and effects of local chemical environment are important, they cannot be treated explicitly within the single-site approximation, on which the CPA is based. Furthermore, despite the fact that Taylor formulated the CPA for the lattice dynamics problem [73], we are not aware of a broad application of the methodology in practice. Thus, a simultaneous treatment of magnetic excitations and lattice vibrations is hardly feasible at present. Thus, approaches that go beyond the CPA are highly requested. 


\subsection{Supercell approach}

Following [72], let us start with the classical Heisenberg Hamiltonian (9) and introduce the average spin-spin correlation functions for coordination shell $\alpha$ :

$\left\langle\Phi_{\alpha}\right\rangle=\frac{1}{N} \sum_{i, j \in \alpha} \mathbf{e}_{\mathbf{e}} \mathbf{e}_{j}$,

where $N$ is the number of atoms in the system. In this formalism, the energy of a magnetic state can be written as:

$$
E_{\text {magn }}=-\sum_{\alpha} J_{\alpha} n_{\alpha}\left\langle\Phi_{\alpha}\right\rangle
$$

where $J_{\alpha}=J_{0 j}$ for $j \in \alpha, n_{\alpha}$ is the number of atoms in the $\alpha$-th coordination shell on the lattice. For example, one can immediately see that the magnetic contribution to the energy of the ferromagnetic state $E_{F M}=-\sum_{\alpha} J_{\alpha}$, while for the fully disordered paramagnetic state $E_{P M}=0$, because in the high-temperature paramagnetic state with the complete disorder between the local moments the average spin-spin correlation functions fulfil the condition

$$
\left\langle\Phi_{\alpha}\right\rangle=0 \quad \forall \alpha \quad, \quad(14)
$$

One way to fulfil Eq. (14) in first-principles simulations is to employ the idea of the so-called special quasirandom structures (SQS) put forward by Zunger et al. to describe chemically disordered alloys [74], and generalized for the case of magnetic disorder by Alling et al. [72]. Because the exchange parameters should decay with distance, one assumes the finite range of the interactions, which simplifies condition (14): $\left\langle\Phi_{\alpha}\right\rangle=0$ only for $\alpha$ with $J_{\alpha} \neq 0$. Indeed, if $J_{\alpha}=0$, any value of $\left\langle\Phi_{\alpha}\right\rangle$ does not influence the left-hand side of Eq. (13). Note that while the original condition (14) requires to dealing with in principal infinite system, the SQS condition can be fulfilled in the finite size periodic supercell. Moreover, as has been emphasized in Sec. 5.1, for the ideal paramagnetic state only collinear spin configurations should in principal be sufficient. The formalism is easily generalized to include multi-site interactions [72]. Of course, in using magnetic SQS one should have in mind all the limitations of the conventional SQS methodology for the treatment of configurational disorder, reviewed in details in Ref. [36].

Moreover, as pointed out by Ruban and Razumovskiy [75], if one deals with an ideal Heisenberg model system, the magnetic interactions are constant, and the way condition (14) is satisfied does not matter. Therefore, one can perform averaging over a proper set of magnetic systems. Every member of such a set can have an arbitrary magnetic structure, but their average should fulfil Eq. (14). One method that achieves this goal is the magnetic sampling method (MSM) suggested by 
Alling et al. [72]. In the MSM, one generates a set of magnetic distributions with random orientation of local magnetic moments, though without taking care of the obtained correlation functions, in contrast to the SQS approach. This can be easily done, for instance, using a random number generator. Then the electronic structure calculations are carried out for each configuration, and the average of the obtained total energies is taken as the potential energy of the paramagnetic sample. In Ref. [72] it was shown that for paramagnetic CrN with B1 crystal structure MSM calculations converged already at 40 different magnetic distributions. Moreover, three different approaches, the DLM-CPA, the magnetic SQS and the MSM gave almost identical results for the potential energy of this system.

It is important to underline that because the goal of the supercell calculations is most often to approximate the potential energy $\left\langle E_{P M}\right\rangle$ of the paramagnetic alloy at high temperature $T$, strictly speaking it should be defined as a thermal average over the 'fast' magnetic degrees of freedom [76]:

$$
\left\langle E_{P M}\right\rangle=-\frac{\partial \ln (Z)}{\partial \beta}=\sum_{\sigma} P_{\sigma} E_{\sigma}
$$

where $Z=\sum_{\sigma} Z_{\sigma}=\sum_{\sigma} g_{\sigma} \exp \left\{-\beta E_{\sigma}\right\}$ is the canonical partition function, $P_{\sigma}$ is the thermal probability of a particular (magnetic) configuration $\sigma, \beta=\frac{1}{k_{B} T}$, where $k_{B}$ is the Boltzmann's constant. $E_{\sigma}$ and $g_{\sigma}$ are the energy (per atom) and the multiplicity of each magnetic configuration, respectively. In fact, using Eq. (15) one can check whether the DLM approach is adequate for the problem at hand or not. Indeed, if the condition for the applicability of the DLM $T \gg J_{i j}^{\max }$ is fulfilled the potential energy calculated from Eq. (15) should be in good agreement with the result obtained by an arithmetic average over different magnetic configurations. For example, in the case of substitutional (N, V) and interstitial (C, N) impurities in $f c c \mathrm{Fe}$ Ponomareva et al. have found negligible difference between the two averaging methods at the temperatures corresponding to the stability field of austenite [76], which has justified the use of the DLM picture in that study.

Ruban and Razumovskiy [75] also pointed out that one has to be careful using the supercell approach because it provides a DLM-like distribution of the magnetic moments only on average for the whole supercell, while specific local correlation functions are quite arbitrary. In the case of modeling of local defects, such as vacancies, impurities, surfaces, interfaces, one therefore has to perform the corresponding averaging over magnetic configurations locally. Consider, for 
(a)

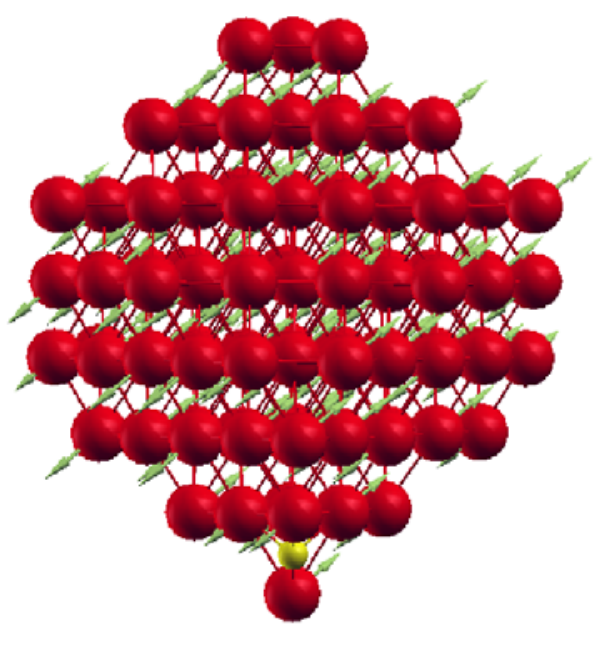

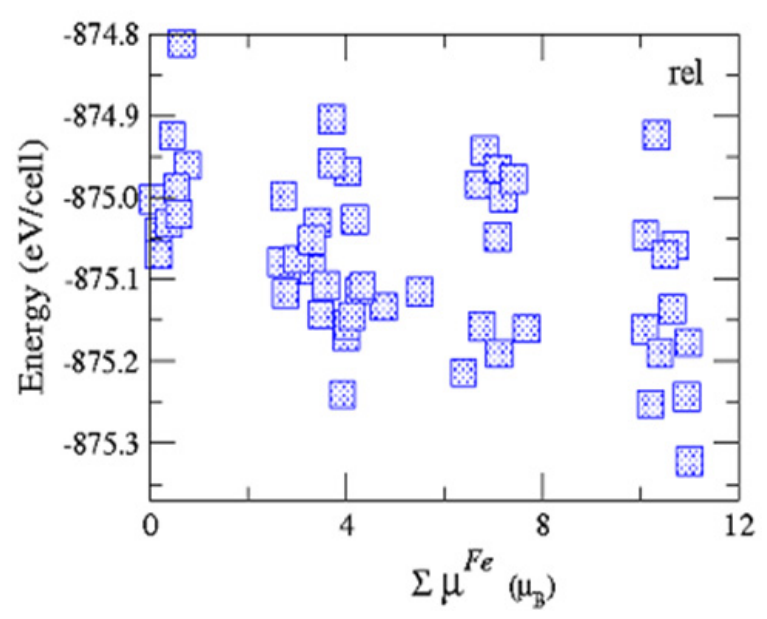

Figure 4. (a) Supercell realization of the disordered local moment model for calculations of the solution energy of an interstial impurity, like C (small yellow atom) in paramagnetic fcc Fe (large red atoms). (b) Using a combination of magnetic special quasirandom structure and magnetic sampling methods, one calculates energies of supercells with different impurity positions in the magnetic SQS representing the host, which are shown here versus the total magnetic moment of Fe atoms located in the first coordination shell of the impurity. Significant variation of the calculated energies depending on the impurity positions in the supercell is clearly seen in the figure.

instance a straightforward use of the magnetic SQS approach for the calculations of impurity solution energies. By placing the impurity at one particular position in the supercell representing the host, the former will sense only one magnetic configuration (Fig. 4a). Indeed, the magnetic SQS approach gives a static picture of the "frozen" magnetic disordered and does not account for the spin dynamics. Note that atomic diffusion processes are several orders of magnitude slower than the magnetic degrees of freedom, therefore on the time scale associated with the diffusion magnetic fluctuations are almost instant. This means that the impurity will sense many different magnetic configurations rather than only one.

Ponomareva et al. [76] has addressed this issue and proposed a combination of the magnetic SQS and MSM approaches for calculations of point defects in paramagnetic hosts. To catch the dynamic behavior of the magnetic system Ponomareva et al. approximated the paramagnetic material by a set of many magnetic configurations, following the idea of the MSM. However, they combined it with magnetic SQS method, which was used to represent the host and to allow for an accurate description of small energy differences between the systems with and without the impurity. The proposed MSM-SQS scheme for calculation of solution enthalpies is as follow. First, one uses the magnetic SQS method to simulate the paramagnetic host. Next one varies 
magnetic distributions around the impurity by changing its positions inside the magnetic SQS and calculates the total energies, which depend strongly on the impurity position (Fig. 4b). Then one averages the energies using Eq. (15) and obtains the potential energy of the paramagnetic system with the defect. If the convergence with respect to the SQS size is achieved, the magnetic SQS for the host with or without the defect ensures that Eq. (14) is fulfilled globally, while MSM sampling of the position of the defect inside the SQS ensures the fulfillment of Eq. (14) for the average local spin-spin correlation functions on the defect site.

In using the supercell realizations of the DLM picture, one should keep in mind that the random distribution of spins on the underlying lattice does not guarantee that the result of the selfconsistent calculations corresponds to the ideal DLM state [75]. In the DLM it is assumed that all the local magnetic moments for chemically equivalent atoms have exactly the same magnitude. This is usually not the case for the systems with the itinerant type of magnetism treated within the supercell approach. Another important issue is related to calculations of interatomic forces and a possibility to account for the local lattice distortions, e.g. around the defects. In fact, this is one of the main motivations for the use of the supercell approach rather than the DLM-CPA scheme.

Unfortunately, fixing a magnetic state in time would lead to artificial static displacements of atoms off their lattice sites due to forces between the atoms with different orientations of their local moments and with different local magnetic environments. In the case of $\mathrm{CrN}$ this was clearly demonstrated in Ref. [27]. On the other hand, in a real paramagnet the fluctuations of the local moments in time should average out and suppress this effect, at least partially. The existence of the local lattice relaxations for the ideal crystal with chemically equivalent atoms at high symmetry positions is an obvious artifact of the model that describes high-temperature state with fluctuating spin fields and moving atoms in a static picture suitable for conventional electronic structure calculations at $T=0 \mathrm{~K}$. It can have significant effect on the calculated potential energy. Figure 5 illustrates this for the case of CrN. The static relaxation energy of the fixed magnetic SQS (circles) is quite large - $0.036 \mathrm{eV} / \mathrm{f}$.u., and unphysical. This is a clear signature of the brake-down of an attempt to model a space and time fluctuations with a purely spatial disorder. However, the artificial effects of the local lattice relaxations can cancel each other in calculations where potential energies of two paramagnetic systems have to be compared, for instance in calculations of the impurity solution energies or mixing enthalpies of the paramagnetic alloys [77]. 


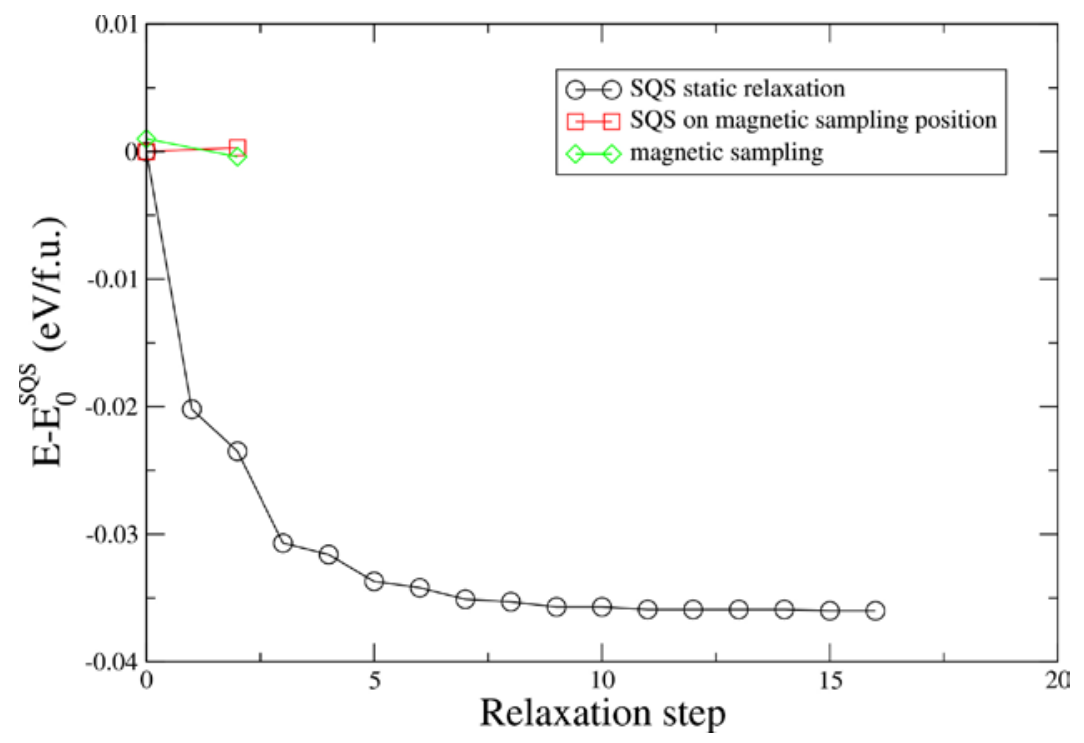

Figure 5. (Color online) Relaxation energies (in eV per f.u.) in the 64-atom $\mathrm{CrN}$ supercell as a function of relaxation iteration step. The spurious and relatively large static relaxation energies of the fixed magnetic SQS configuration (black circles) is contrasted with the relaxation energy of the magnetic sampling method (green diamonds) which is nearly vanishing within the accuracy of the method. Here a series of magnetic samples is considered and the forces acting on each nucleus in all the samples are averaged. This average force is then used to guide the nuclei to their optimal static positions. Also the energy of the SQS configuration calculated on the structure obtained with the magnetic sampling method is shown for comparison (red squares). Here we perform one small relaxation step in all the calculations of the magnetic samples. Then we put together the new positions and average them, resulting in the actual relevant new positions. The agreement between the two later methods with magnetic SQS calculations without local lattice relaxations underlines artificial nature of the conventional static approach. Details of calculations are the same as in Ref. [72].

\subsection{Spin wave method}

Besides the above-mentioned complications of the supercell approach, it becomes quite time consuming in the case of random alloys or large and inhomogeneous systems. Ruban and Razumovskiy have proposed another scheme that fulfills condition (14), the spin wave method (SWM) [75]. They considered a set of the planar spin spirals with the azimuth angle $\pi / 2$ for all the wave vectors $\mathbf{q}$ in the corresponding Brillouin zone and showed that the spin-spin correlation function for a superposition of all the spin spirals with different wave vectors averages to zero, thus fulfilling Eq. (14). The energy of the paramagnetic state in the SWM is given by the integral over the Brillouin zone of the energies of each planar spin spiral with the wave vector $\mathbf{q}$. In practice, one does this using the special point technique, requiring to carry out DFT calculations for a finite set of $\mathbf{q}$-vectors. 
In application of the SWM method for calculating properties of Fe and Co, the results were found to be very close to those obtained by means of the DLM-CPA calculations. However, the former can be combined with the supercell geometry, for example to describe defects or local relaxations in chemically disordered systems. In particular, the SWM allows one to calculate the defectformation energies, elastic constants [75], and phonon spectra [78]. One of the advantages of the spin-wave method is a possibility to model a magnetic state with specific magnetic short-range order, the effect, which is difficult or impossible to study with other approaches described above. Of course, the method describes the high-temperature paramagnetic state as a set of noninteracting magnons. This can be questioned from the fundamental point of view. On the other hand, semi-classical spin-dynamics simulations using Eq. $(6,7,9)$ carried out to elucidate a longstanding controversy regarding the existence, or otherwise, of spin waves in paramagnetic bcc iron, point to their existence [53]. In addition, one has to remember that the justification of the SWM involves the independence of the exchange parameters on the magnetic state, which is not the case in many itinerant systems. One should bear in mind, however that the SWM method gives the field very new and interesting idea. Its evaluation in future applications will contribute to better understanding of its advantages and limitations.

\section{Lattice dynamics of paramagnetic materials.}

In most applications, the simulations of the magnetic materials in the paramagnetic state are still carried out at the fixed crystal lattice, neglecting contributions from lattice vibrations, which ought to be increasingly important when one goes from low-temperature magnetically ordered state to the high-temperature paramagnetic state. As has been pointed out in Sec. 3, the LDA+DMFT calculations are too time consuming. Moreover, at present forces within the LDA+DMFT approach have only been calculated directly from the derivative of the total energy. In particular, this approach was employed by Leonov et al. [44] in the calculations of the phonon dispersion relations for Fe. Leonov et al. computed the lattice dynamics of paramagnetic iron using the GGA + DMFT approach in combination with the method of frozen phonons. The phonon frequencies were calculated by introducing a small set of displacements in the corresponding supercells of the equilibrium lattice, which results in a total energy difference with respect to the undistorted structure. Unfortunately, computational costs associated with these types of calculations are very high at present. This makes it difficult to efficiently couple magnetic and vibrational degrees of freedom within the DMFT calculations in a simultaneous treatment of molecular and spin dynamics, needed for a description of materials behavior at high temperature and fro calculations of all free energy contributions to Eq. (1) at the same footing, as discussed in Sec. 2. 


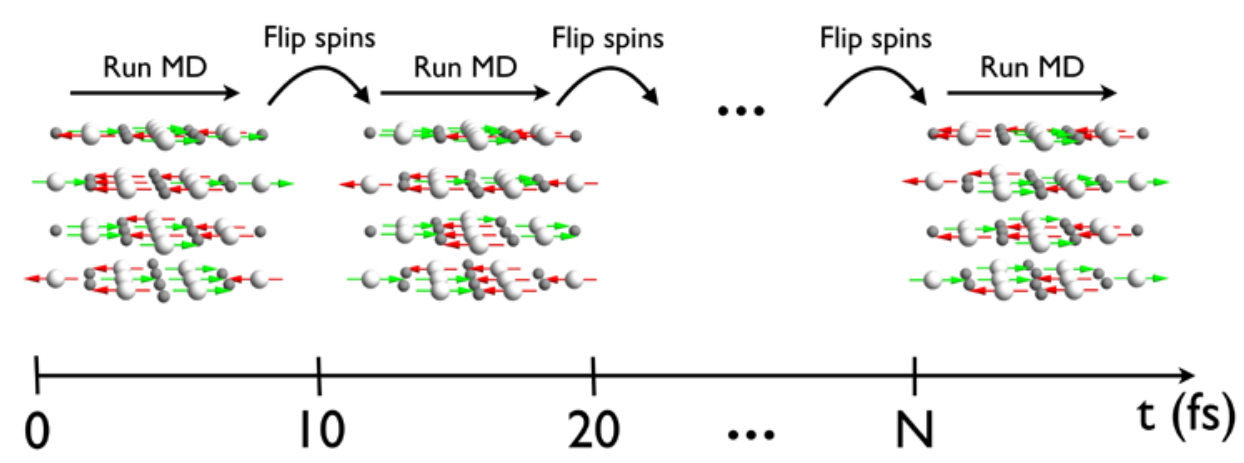

Figure 6. (Color online) Schematic illustration of DLM-MD algorithm. See text for more discussion.

Theoretical formalism that combines quasi-classical spin dynamics with molecular dynamics was presented in Ref. [10]. However, it is too time consuming and to the best of our knowledge, it is not realized in practice yet. A combination of semi-classical spin dynamics and molecular dynamics should be more numerically efficient, and there is a significant progress in this direction [79,80]. For example, Perera et al. [81] have simulated properties of bcc Fe at room temperature, while Tao et al. [53] have studied its spin dynamics above $T_{C}$. However, in addition to the problems of the semi-classical approach discussed in Sec. 3, one would have to treat a dependence of effective exchange parameters on the distances between atoms. The latter may be significant $[58,82]$. Thus, more approximate solutions to the problem are sought.

\subsection{Disordered local moment molecular dynamics.}

Steneteg et al. [27] suggested to implementing the DLM picture in the framework of $a b$ initio molecular dynamics (MD) within the DLM-MD method. DLM-MD algorithm shown in Fig. 6 realizes numerically the concept of of temporarily broken ergodicity [25] discussed in Sec. 5.1. According to it the magnetic subsistem gets stuck for time $t_{S F}$ at a point of the phase space corresponding to the particular magnetic configuration and then moves rapidly to another point. Remember that $t_{S F}$ denotes the time between spin-flips which is long enough (in the particular case of Fig. 6 it corresponds to $10 \mathrm{fs}$ ). Steneteg et al. made an approximation that the magnetic state of the system is completely randomly rearranged with a time step $t_{S F}$, and with a constraint that the net magnetization of the system should be zero. To simulate a paramagnetic system, they initialize calculations by setting up a supercell where collinear local moments are randomly oriented and the total moment of the supercell is zero. Then they run collinear spin-polarized MD for the number of MD time steps. Denoting the MD time step as $t_{M D}$ and requiring that $t_{M D}<<$ $t_{S F}$, the number of the MD time steps that the system stays in the same magnetic state is given by $N_{M D}^{S F}=t_{S F} / t_{M D}$. During the run, the magnitudes of the local magnetic moments are allowed to 
vary as dictated by the self-consistent solution of the electronic structure problem at each step of the MD simulation. In principle, the orientations of the magnetic moments are not restricted, so they are allowed to flip as well. Thus, the net magnetic moment in the simulation cell should be checked to make sure that the system remains in the DLM state. When $N_{M D}^{S F}$ steps of the MD are executed the spin state is randomized again by setting up another configuration of disordered local moments, while the lattice positions and velocities are unchanged, and the simulation run continues (Fig. 6). In several applications of the DLM-MD [27, 77] it was demonstrated that the algorithm depicted in Fig. 6 was numerically stable and led to well-converged values of observable properties, like the potential energy.

Of course, $t_{S F}$ remains the model parameter, which cannot be determined self-consistently within DLM-MD simulations. In fact, spin-lattice dynamics simulations for ferromagnetic bcc Fe at 300 $\mathrm{K}$ [79] demonstrated that the characteristic time scale for the quasiperiodic motion of an atom is of the order of $0.1 \mathrm{ps}$ (which is the inverse Debye frequency of the material). The time scale that characterizes the dynamics of precession of atomic spins is significantly shorter, of the order of fs. Thus, following arguments given in Sec. 2, $t_{S F}$ value of the order of 10 fs should be reasonable, though it might be system-dependent.

As a matter of fact, Steneteg et al. conducted an additional numerical experiment that allowed them to argue for their choice of $t_{S F}$ (in Ref. [27] DLM-MD calculations were carried out for paramagnetic cubic B1 phase of $\mathrm{CrN}$ ). They analyzed the $\mathrm{Cr}-\mathrm{Cr}$ metal nearest neighbor distances, and separated them into pairs with parallel and antiparallel orientations of the local moments. Thus, the effect of $t_{S F}$ on the distribution of pair distances became apparent. If $t_{S F}$ was very short, $10 \mathrm{fs}$, there were no noticeable difference between the two sets of pairs, indicating that the atoms did not have time to adjust their positions for the current orientation of local magnetic moments. If $t_{S F}$ was increased to $100 \mathrm{fs,} \mathrm{the} \mathrm{atoms} \mathrm{had} \mathrm{sufficient} \mathrm{time} \mathrm{to} \mathrm{move} \mathrm{towards}$ the energetically more favorable positions, leading to an observable shift in distances between pairs with parallel and antiparallel local moments. We could not rule out a possibility of statistical correlations between the atomic distances and the orientation of atomic moments in a dynamically changing paramagnetic phase. However, to the best of our knowledge it has never been reported in experiments. Moreover, its appearance would be contra-intuitive. Therefore, Steneteg et al. argued [27] that in practical applications of the DLM-MD one should use smaller values of $t_{S F}$, that would ensure the absence of differences in distances between atoms with parallel and antiparallel local moments. Importantly, they also observed that the potential energy shift obtained in the DLM-MD simulations for paramagnetic cubic CrN was $\sim 1$ meV/f.u. for $t_{S F}$ less than $15 \mathrm{fs}$, while it increased substantially for the larger values $t_{S F}$. The presence of an energy plateau on the potential energy vs $t_{S F}$ curve may justify further the choice of short spinflip times in DLM-MD simulations. In fact, it indicated that one could use the adiabatic approximation and to consider spin-flips as fast degrees of freedom with respect to atomic motion. The adiabatic approximation could significantly simplify the simulations of lattice dynamics in paramagnetic materials, as will be discussed in Sec. 6.2. 
Shulumba et al. [83] used the DLM-MD approach to develop a framework for calculation of the Gibbs free energy of a paramagnetic material, Eq. (1), which coupled all the terms, $G_{e l}$, $G_{v i b}$, and $G_{\text {magn }}$ to each other and treated them simultaneously in first-principles calculations. Shulumba et $a l$. has combined the DLM-MD simulations of disordered magnetism with the temperaturedependent effective potential method [84] to obtain the vibrational contribution to the free energy. This has made it possible to model the phase stability of a magnetic material in its hightemperature paramagnetic phase, including temperature-induced anharmonic and harmonic vibrational, as well as the magnetic effects simultaneously. Shulumba et al. have applied the technique to investigate the phase diagram of $\mathrm{CrN}$ and have found that the vibrational contribution have favored the stability of the cubic paramagnetic phase with respect to the orthorhombic antiferromagnetic phase. The predicted temperature for the transition has been lowered as compared to the static calculations, bringing it in better agreement with experiments.

Once again, here we deal with a new methodology, and its evaluation should take some time. Major approximations in the present realization of the DLM-MD method, which are likely to introduce inaccuracies, include the usage of collinear moments, the temporarily broken ergodicity of the DLM approach, and the existence of the parameter $t_{S F}$. In addition, so far the scheme has been applied in simulations of very good Heisenberg systems.

However, our recent results indicate that also more itinerant systems, like fcc-Fe can be addressed with DLM-MD. Figure 7 shows a histogram of resulting values of local Fe magnetic moments during several pico-seconds run of DLM-MD simulations of fcc-Fe at $1662 \mathrm{~K}$, a temperature just below the gamma-to-delta transition. The figure shows that the vast majority of Fe local moments have magnitudes around $2 \mu_{B}$, but they point into positive or negative directions depending on their initial orientation. Nevertheless, these results illustrate that there is a considerable variation in magnitudes of the moments. This variation is not related to the longitudinal spin fluctuations discussed in Secs. 2 and 4, but is instead the direct impact of atomic vibrations, as well as sensitivity of magnetic interactions to the local magnetic environments. Thus, the result shown in Fig. 7 explicitly demonstrates that lattice vibrations and local environment effects have to be considered in a quantitative description of the paramagnetic state of itinerant magnets. Clearly, the DLM-MD technique has high potential to become a valuable tool for studies of magnetic materials at high temperature. 


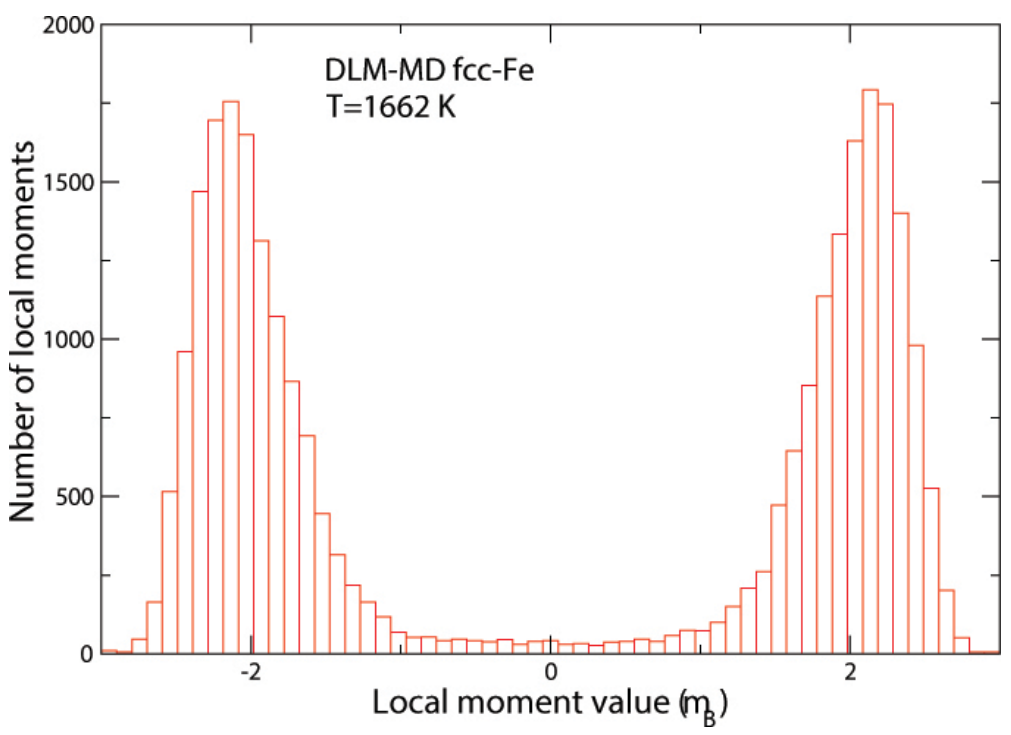

Figure 7. Histogram of resulting values of local magnetic moments during several pico-seconds run of DLM-MD simulations of fcc-Fe at $1662 \mathrm{~K}$.

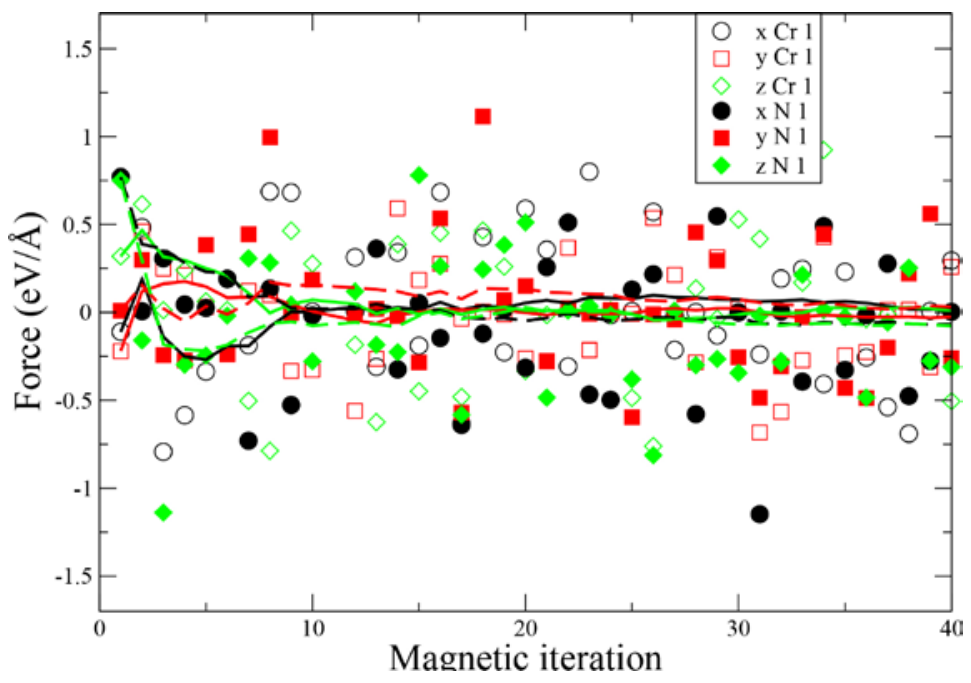

Figure 8. (Color online) The Hellmann-Feynman forces in $\hat{x}, \hat{y}$, and $\hat{z}$ direction acting on a $\mathrm{Cr}$ atom (open symbols) and a N-atom (solid symbols) for each MSM configuration of the supercell. The lines indicate the accumulated average of the forces over MSM configurations in each direction for the Cr-atom (solid lines) and N-atom (dashed line). Details of calculations are the same as in Ref.[72]. 


\subsection{Averaging of interatomic forces in the adiabatic limit.}

In the adiabatic limit, qualitatively justified in Sec. 6.1, the magnetic degree of freedom is fast with respect to lattice vibrations. This means that the instantaneous forces acting on the atomic nuclei will change rapidly in the paramagnetic state of the system, on the same time-scale as the fluctuations of the local orientations of the magnetic moments. Thus, the relevant forces that determine lattice dynamics are not the instantaneous forces of any particular magnetic configuration. Instead, a better approximation for the lattice dynamics should use the forces averaged over the magnetic fluctuations to govern the nuclei in its motion. In particular, in the adiabatic approximation for the paramagnetic state the fluctuations of the magnetic configurations are much faster than the relaxation of the nuclei to their equilibrium positions. Thus, the forces acting on each nucleus (the forces averaged over the magnetic fluctuations on the time scale relevant for the lattice dynamics) can be approximated with the forces averaged over different magnetic configurations.

To illustrate this concept we consider the case of paramagnetic B1 CrN without defects. In this system, due to symmetry, there should not be any static lattice displacements of the atoms away from the ideal lattice points. This means that the forces averaged over the magnetic fluctuations on the time scale relevant for the lattice dynamics should be zero. Figure 8 shows calculated Hellmann-Feynman forces acting on a $\mathrm{Cr}$ and a $\mathrm{N}$ atoms placed on ideal B1 lattice positions in the supercells with different magnetic configurations, generated in the framework of MSM simulations (Sec. 5.2).

Typically, the forces are rather large reflecting the magnetic strain effects in CrN discussed in Ref. [85] and the low symmetry of each structure induced by the magnetic disorder. However, considering the average force of a series of MSM samples, the situation is very different. In fact, the average forces are converging towards zero already after 40 iterations, which coincides with the convergence of the potential energy for this system [72]. This means that the cubic symmetry of $\mathrm{B} 1 \mathrm{CrN}$ is retained only in calculations that use the average forces.

Thus, the following strategy for calculating forces acting on individual atoms in the paramagnetic state of magnetic materials can be used in the adiabatic limit. A series of magnetic configurations are considered and the forces acting on each nucleus in all the samples are averaged. Then this average force is used to determine the dynamics of nuclei in MD or phonon calculations. In fact, Ruban and Razumovskiy [75] have used this strategy in calculations of phonon dispersion relations in paramagnetic bcc Fe by means of the SWM. Körmann et al. [86,87] employed it in the spin-space averaging method (SSA) coupled to the magnetic SQS method for simulations of lattice dynamics in $b c c$ and $f c c$ Fe by using. A justification of the adiabatic approximation presented here provides a support to these works. 


\section{Overview of recent applications.}

The development of techniques that allow one to simulate magnetic materials in their paramagnetic state, reviewed in Secs. 3-6, together with the significance of these materials for fundamental studies and technological applications stimulated enormous research activity. Here we give an overview of selected recent applications, without claiming its completeness, which is hardly feasible in this rapidly developing field of research. For earlier applications we refer to the recent papers discussed below, which generally contain high-quality literature overviews. Moreover, we are mostly interested in simulations that considered effects of temperature induced magnetic disorder rather than treated studied systems either as magnetically ordered or as nonmagnetic. In addition, in most cases, except Sec. 7.4, we are interested in simulations of thermodynamic and mechanical properties, rather than in the studies of the electronic structure and magnetic phase transitions.

\subsection{Transition metals.}

Iron represents the base metal for steels, and its paramagnetic state has been in focus of intense research. It is the most abundant element on our planet. It is one of the most important technological materials and, at the same time, one of the most challenging elements for the modern theory. The ground state of Fe at ambient conditions, the $\alpha$-phases (ferrite, $b c c$ ) is ferromagnetic, with a Curie temperature $T_{C} \sim 1043 \mathrm{~K}$. The $\gamma$ phases (austenite, $f(c)$ ) is stabilized at $\sim 1185 \mathrm{~K}$, but upon further heating up to $\sim 1670 \mathrm{~K}$ the $b c c$ structure reappears again ( $\delta$-iron). All phases of Fe are paramagnetic above the $b c c T_{C}$, and therefore the description of the structural phase transitions in Fe requires a careful treatment of the magnetic disorder. To make the problem even more complicated, the accuracy needed for the description of the phase diagram of Fe should be $\sim 1 \mathrm{meV}$ [88].

While it is broadly accepted that the bcc Fe can be characterized as a system with well-defined local magnetic moments in the paramagnetic state [23,37], the situation on the $f c c$-Fe is less clear. Igoshev et al. [42] have applied the LDA+DMFT approach to the paramagnetic $\gamma$-iron and have revisited the problem of the theoretical description of its magnetic properties in a wide temperature range. Their results show that at low temperatures $\gamma$-iron is better described in terms of the itinerant picture. At the same time, in the temperature range $T=1200-1500 \mathrm{~K}$, corresponding to the stability field of the $f c c$ phase of iron, it can be characterized by temperature-dependent effective local moments, which yield relatively narrow peaks in the real part of the local magnetic susceptibility, Eq. (3), as a function of frequency. In particular, in this temperature window inverse susceptibility has depended nearly linearly on the temperature, following the Curie-Weiss law. 
Leonov et al. [43] underlined the importance of correlation effects for the description of the $\alpha$ - to $\gamma$-phase transition in Fe at elevated temperature and ambient pressure. Considering the variation of the total energy of paramagnetic iron with temperature along the $b c c-f c c$ Bain transformation path, Leonov et al. have observed the energetic stabilization of the latter with respect to the former at reduced temperature $T \sim 1.3 T_{C}$. The authors emphasize that the difference between the temperatures at which the magnetic transition and the vanishing of the structural energy differences between the two phases occurs is in remarkable agreement with the experimental difference in temperatures between magnetic and structural phase transitions in Fe, that is $~ 200$ K. However, Belozerov et al. [89] pointed out that the agreement was achieved only in terms of the reduced temperature $T / T_{C}$, while the calculated Curie temperature $T_{C}$ was found to be significantly larger than the experimental value of $1043 \mathrm{~K}$. In fact, Belozerov et al. showed that if quantum Monte-Carlo calculations within DMFT were limited to the Ising-type exchange interaction, the calculated Curie temperature of Fe should be overestimated almost by factor 2 . They proposed the rotationally invariant Hirsch-Fye quantum Monte Carlo algorithm that reduced calculated $T_{\mathrm{C}}$ in $b c c \mathrm{Fe}$ to $\sim 1260 \mathrm{~K}$, bringing it in better agreement with the experiment. However, to the best of our knowledge the importance of this modification for the description of structural properties of Fe has not been reported yet.

Several concerns with the LDA+DMFT calculations for Fe have been put forward in the literature [90]. In particular, they have shown the increasing instability of the $b c c$ lattice with temperature with respect to the $f c c$ lattice, which would make it difficult to explain $\gamma$ to $\delta$ transition in iron. Moreover, the $f c c-b c c$ structural energy difference have converged towards the value obtained from $0 \mathrm{~K}$ nonmagnetic calculations, and the $b c c$ structure at high temperature ( $T$ $>1.3 T_{\mathrm{C}}$ ) has shown the mechanical instability. In fact, DFT calculations by Okatov et al. [91] using magnetic SQS also predicted significantly higher stability of the paramagnetic $f c c$ Fe with respect to its paramagnetic bcc phase, and mechanical instability of the latter. DLM-CPA DFT calculations by Zhang et al. [90], on the contrary, showed that the paramagnetic bcc Fe should be mechanically stable, while the energy difference between the two phases should depend strongly on their lattice parameters. However, Ruban and Razumovskiy [75] showed that the mechanical stabilization of the paramagnetic bcc Fe obtained by Zhang et al. could be an artefact of the use of spherical approximation for the one-electron potential in Ref.[90].

Unfortunately, the LDA+DMFT calculations in Ref. [43], as well as magnetic SQS calculations by Okatov et al. [91] and DLM-CPA calculations by Zhang et al. [90] focused exclusively on the total energy differences between competing structures of Fe. On the other hand, the importance of vibrational contribution to the free energy is well recognized in the field. In particular, Körmann et al. [92] calculated thermodynamic properties of ferromagnetic bcc iron up to the bcc$f c c$ phase transition temperature including vibrational, electronic, and magnetic contributions at the GGA-DFT level. An excellent agreement was obtained with available experimental data, but 
only when all three types of excitations were included. Note, however, that the adiabatic decoupling between vibrational, electronic, and magnetic contributions to the free energy was employed in Ref. [92]. In particular, phonon dispersion relations were calculated for ferromagnetic $\mathrm{Fe}$ at $\mathrm{T}=0 \mathrm{~K}$, and used at high temperature, neglecting the modification of the magnetic state by temperature induced excitations. In the subsequent work by Körmann et al. [86] the authors demonstrated that in the case of bcc iron the longitudinal branches obtained in phonon calculations assuming the ferromagnetic state were in reasonable agreement with experiment. On the contrary, for paramagnetic bcc Fe the experimentally observed pronounced softening at high temperatures, in particular of the transversal modes between $H$ and $P$ points in the Brillouin zone, as well as between $\Gamma$ and $N$ points were not reproduced.

Lavrentiev et al. [34] employed the magnetic cluster expansion, Eq. (10) to model bcc-fcc transitions in Fe, including both, the $\alpha-\gamma$ and $\gamma-\delta$ phase transitions. Their calculations demonstrated that both the magnetic and phonon excitations contribute to the free energies of the $\alpha, \gamma$ and $\delta$ phases. Unfortunately, the approach was not fully ab initio. In particular, the phonon contribution to the free energy of bcc $\alpha$-iron was derived from experimental elastic constants measured for the highest available temperature, $1173 \mathrm{~K}$, at which $b c c$ iron remained mechanically stable. For $f c c \gamma$ iron Lavrentiev et al. used a force-constants model derived from experimental data on inelastic neutron scattering at $1428 \mathrm{~K}$.

Ma and Dudarev [80] performed the spin-lattice-dynamics simulations of the magnetocaloric effect in bcc Fe (and hcp Gd), with the Heisenberg and Landau parameters of Hamiltonian (10) of interacting atomic magnetic moments derived from $a b$ initio simulations. The simulations described the complete thermodynamic cycles involving dynamic adiabatic magnetization, isofield thermalization, adiabatic demagnetization, and isofield thermalization, and the microscopic equilibrium and nonequilibrium relaxation aspects of the magnetocaloric phenomenon.

The importance of vibrational contribution in simulations of the temperature induced phase transitions in Fe stimulated several groups to calculate the phonon dispersion relations for paramagnetic $b c c$ and $f c c$ Fe from first principles. Leonov et al. [44] carried out GGA+DMFT calculations of the phonon dispersion relations and obtained surprisingly good agreement with experiment for the both, $b c c$ and $f_{c c}$ Fe at reduced temperatures $1.2 T_{C}$ and $1.4 T_{C}$, respectively. Both phases of Fe were found to be dynamically stable at their respective temperatures. In the $b c c$ Fe Leonov et al. observed a weak anomaly in the transverse $T_{1}$ acoustic mode along the [ $\xi \xi 0$ ] direction, indicating that at $T \sim 1.2 T_{C}$ the $b c c$ phase may be close to an instability, and ascribed this result to a dynamical precursor effect of the bcc-to-fcc phase transition. 
Interestingly, much simpler calculations of Körmann et al. [86] in the framework of GGA-DFT using the SSA method and the averaging of the interatomic forces in the adiabatic limit reproduced very well the DMFT calculations of Leonov et al.[44], as well as the experiment. In particular, for the $b c c$ Fe the strong softening of the transversal $\Gamma N$ modes, as well as the softening around the dip in the HP branches were reproduced. Thus, Körmann et al. [86] clearly demonstrated the large impact of magnetic disorder on the vibrational properties of $b c c$ iron. Ruban and Razumovskiy [75] calculated the phonon dispersion relations for the $b c c$ Fe in the paramagnetic phase using the SWM [75]. The agreement between the SWM results, DMFT calculations [44] and experiment was good [78], except the $T_{1}$ mode in the $\Gamma-N$ direction for which two of the three chosen spin-wave sets yielded negative energy indicating the dynamical instability of the considered phase. Ruban and Razumovskiy argued that magnetic short-range order above $T_{C}$ should be taken into account to correctly describe lattice dynamics of the paramagnetic $b c c$ Fe. In a subsequent study Körmann et al. [87] proposed a simplified scheme for the interpolation of forces calculated in the ferromagnetic and paramagnetic states (by means of the SSA) to account for the effects of magnetic short-range order. They confirmed the strong impact of the latter on the phonon dispersion relations in bcc Fe even significantly above the Curie temperature.

Turning to the problem of phase stability of Fe upon compression, the $b c c$ Fe is transformed into the hcp phase ( $\varepsilon$-iron) above $\sim 12 \mathrm{GPa}$. The magnetic state of the latter is still debated [93]. While numerous DFT calculations require complex magnetic order in the $h c p$ Fe to explain its lattice parameters and bulk modulus, the experimental information favors the picture of non-magnetic or paramagnetic $h c p$ phase up to very low temperatures [94]. Using the LDA+DMFT calculations, Pourovskii et al. [95] have demonstrated the possibility to reproduce the properties of the hcp Fe without the requirement of an antiferromagnetic order. Ruban et al. [96] have simulated magnetic properties of the $b c c$ phase of Fe at extreme conditions of the Earth's core, at ultra-high pressure 350 GPa and temperature above 5000-6000 K, while Pourovskii et al. [97] carried out the LDA+DMFT calculations for the $h c p, f_{c c}$ and the $b c c$ phase at the same conditions. Both groups have put forward very interesting possibility of the survival of local magnetic moments in the bcc Fe at the Earth's core conditions. The $b c c$ Fe have been suggested theoretically as a possible stable crystal structure of this metal in the Earth's core [89,99], and some experiments on Febased alloys supported this suggestion [100,101], though the issue is still under debate [102].

As compared to $\mathrm{Fe}$, the paramagnetic state of other magnetic transition metals, $\mathrm{Mn}, \mathrm{Cr}, \mathrm{Co}$, and $\mathrm{Ni}$, is less studied. The temperature dependence of the inverse paramagnetic susceptibility of fcc nickel was calculated by Staunton and Gyorffy using generalized Onsager cavity field in the framework of the disordered local moment picture[29]. Staunton et al. [103] developed a scheme for making $a b$ initio calculations of the dynamic paramagnetic spin susceptibilities of solids at finite temperatures, and studied incommensurate and commensurate antiferromagnetic spin 
fluctuations in paramagnetic $\mathrm{Cr}$, as well as in compositionally disordered $\mathrm{Cr}_{95} \mathrm{~V}_{5}$ and $\mathrm{Cr}_{95} \mathrm{Re}_{5}$ alloys. Belozerov et al. [89] computed the electronic structure and magnetic properties of paramagnetic nickel by employing the LDA+DMFT implemented with rotationally invariant Hirsch-Fye quantum Monte Carlo method. Their comparison of the spin-spin correlation functions between $\alpha$ iron and nickel calculated at $T=2.5 T_{\mathrm{C}}$ showed that the magnetism of nickel is more itinerant. Interestingly, despite this fact, recent experimental and theoretical studies $[104,105]$ demonstrated that the ferromagnetic state of Ni is stable at least up to $260 \mathrm{GPa}$, the highest pressure where magnetism in any material has been observed so far. DMFT calculations carried out by Di Marco et al. [106] for fcc Mn and Ni, pointed out the importance of the correlation effects for the former. Note that Di Marco et al. considered the antiferromagnetic phase of the $f c c$ Mn rather than the paramagnetic phase of this metal.

Körmann et al. calculated various thermodynamic functions, like specific heats and free energies, for $\mathrm{Co}, \mathrm{Ni}$ [107] and $\mathrm{Cr}$ [108], in addition to Fe [107] in the broad temperature interval, including the temperatures above the magnetic transition temperatures. Thermodynamic properties were computed based on quasiharmonic, anharmonic, electronic and magnetic free energy contributions in Eq. (1) calculated from first principles. For the description of magnetic properties, Körmann et al. proposed an approach that mapped the magnetic long-range system onto an effective, nearest-neighbor quantum Heisenberg model, for which the QMC approach provides a numerically exact solution. Of course, serious approximations were introduced. For example, all the terms in Eq. (1) were considered as adiabatically decoupled from each other, meaning that the dependence of lattice vibrations on the magnetic state of the system was neglected. The temperature dependence of local magnetic moments was neglected as well, and the effect of longitudinal spin fluctuations was not taken into account. Still, Körmann et al. obtained encouraging results for the calculated thermodynamic functions $[107,108]$. However, it is clear that more work needs to be done on improving theoretical models for transition metals, like $\mathrm{Cr}$ and $\mathrm{Ni}$, which are more itinerant than $\mathrm{Fe}$.

\subsection{Phase stability of Fe-based alloys.}

Fe-based alloys belong to the most important structural materials. A recent review on advances of density functional theory to finite temperatures and on its applications in steel design can be found in Ref. [109]. Here we concentrate on two systems, Fe-Cr and Fe-Mn based alloys, where the studies of thermodynamic properties in the paramagnetic state are quite intensive.

The binary Fe-Cr alloys are the base for many important industrial steels. The four major types of stainless steel are the austenitic steels with very high corrosion resistance, weldability and ductility, the martensitic steels with high strength and moderate corrosion resistance, the duplex steels characterized by both strength and ductility, and ferritic steels known for their excellent corrosion resistance. For example, Fe-Cr ferritic steels are used to manufacture reactor pressure vessels (RPV). Irradiation-induced accelerated ageing is one of the crucial issues that limits the 


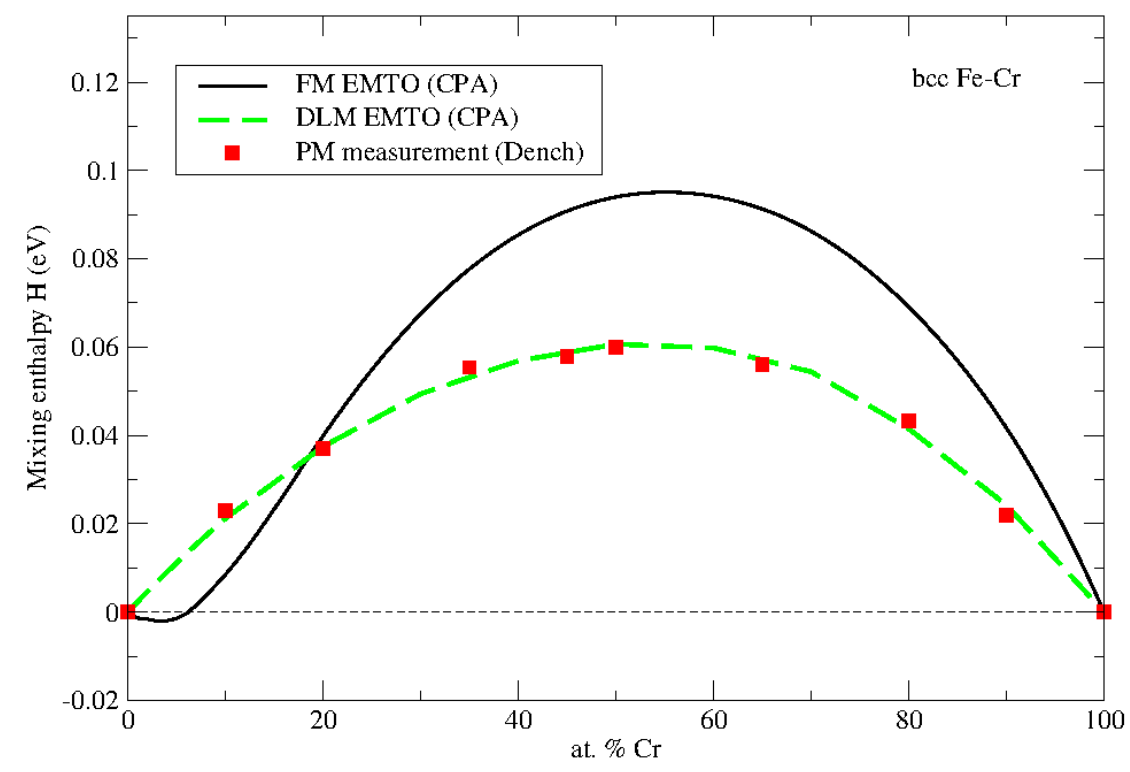

Figure 9. Mixing enthalpies $H$ (in eV/atom) of paramagnetic $b c c$ Fe-Cr alloys simulated by means of DLM-CPA approach (green dashed line). Excellent agreement with experimental data from [112] (red squares) is obtained in DLM calculations. Ferromagnetic results (FM, black solid line) are shown for comparison. Note that FM bcc Fe was used as the standard state in the calculations of the mixing enthalpies of the ferromagnetic alloys, in contrast to DLM bcc Fe, used as the standard state for calculations of the mixing enthalpies in the paramagnetic alloys.

lifetime of nuclear reactors. Fe-Cr steels with 7-18 at. \% $\mathrm{Cr}$ are promising structural materials for fast neutron reactors due to their relatively low rate of swelling at elevated temperatures [110]. In the binary Fe-Cr alloy a spinodal decomposition can lead to a formation of precipitates of $\alpha$, phase, but at low chromium concentrations the alloys are anomalously stable. This effect received significant attention, and motivated large number of studies of mixing thermodynamics of $b c c$ Fe-Cr alloys, following pioneering work by Olsson et al. [111].

In Fig. 9 we show results of our calculations which are in good agreement with those presented in Ref. [111]. Much attention is usually called to a small region of Fe-rich compositions, where the mixing enthalpy, calculated for ferromagnetic $b c c$ Fe-Cr alloys is negative. In view of our focus on the description of the paramagnetic state, we would like to call the attention to an excellent agreement of the mixing enthalpy of bcc Fe-Cr alloys simulated by means of the DLM-CPA method with experimental values measured in the paramagnetic state [112]. The agreement confirms the reliability of the DLM picture for the description of potential energy of magnetic materials in the paramagnetic state. In addition, Fig. 9 underlines the importance to using the 
proper magnetic state for thermodynamics description of magnetic alloys. Indeed, calculated mixing enthalpies differ significantly between ferromagnetic and DLM calculations.

The dependence of chemical interactions on the global magnetic state of the alloy has been emphasized by Ruban et al. in a series of papers dealing with phase stability of Fe-Cr alloys $[113,114,115]$. In fact, this is very general effect [36], importance of which has been demonstrated in other alloys, e.g. in Co-Pt [54], Fe-Ni [20], Fe-Cu [116] and Fe-Pd [117]. Moreover, Ponomareva et al.[118] demonstrated that it should be possible to tune the effective chemical interactions in an alloy by its global magnetic state. The effect allowed the authors to predict theoretically and to synthesize experimentally new cubic $\mathrm{Fe}_{2} \mathrm{Si}$ phase with $B 2$ structure. For Fe-Cr system, Ruban et al. demonstrated [113] that the dependence of chemical interactions on the global magnetic state of the alloy had important implications for phase equilibria: the experimentally reported concentration interval of anomalous ordering in Fe-Cr alloys should be determined by the thermal history of the alloys through the value of global magnetization at the annealing temperature.

In a subsequent work [115], Ruban et al. proposed a model, which includes magnetic, electronic, phonon, and local atomic relaxations contributions to the free energy derived from $a b$ initio calculations, as well as the effects of magnetic short-range-order above $T_{C}$. The approximations here included, for example, a description of finite temperature ferromagnetic state with partial DLM (PDLM) model, that is with non-equal fractions of spin up and spin down atoms. Ruban et al. successfully used this model earlier for the description of Fe-Cr alloys [113], Ekholm et al. employed it for simulations of phase stability in $\mathrm{FeNi}_{3}$ permalloy [20] and Gorbatov et al. [116] in the studies of $\mathrm{Cu}$ precipitation in $\alpha$-Fe. However, one has to be careful with the PDLM approach, as it is limited to the Ising-like collinear description of the partially disordered ferromagnetic state. Non-collinear components, however, may be more important than collinear spin-flip transitions below $T_{C}$ or $T_{N}$ and may dominate the magnetic disorder. Lavrentiev et al. [119] demonstrated this recently by means of the Monte Carlo simulation algorithm based on a Heisenberg-Landau Hamiltonian (10). In addition, the treatment of vibrational contribution in [115] was limited to the ferromagnetic state and to the use of a simplified average force constants model. Despite these limitations, Ruban et al. successfully calculated isostructural phase diagram for the $b c c$ Fe-Cr system, which turned out to be in reasonable agreement with the CALPHAD assessment. In particular, the calculations reproduced the abnormal decrease in the solubility of $\mathrm{Cr}$ in Fe-rich alloys at high temperatures close to the magnetic phase transition, the feature known as a Nishizawa horn, and identified its origin in a relative stabilization of Cr alloying in the ferromagnetic state compared to that in the paramagnetic Fe-rich alloys with up to 20 at.\% Cr. 
Lavrentiev et al. [34] used the magnetic cluster expansion based on the Heisenberg-Landau Hamiltonian (10) in combination with empirical treatment of lattice vibrations, to describe the occurrence of the $\gamma$-loop in the phase diagram of Fe-Cr alloys. The region where $f c c \gamma$-Fe-Cr was found to be more stable than bcc $\alpha$-Fe-Cr extended to $10.5 \% \mathrm{Cr}$, in agreement with the experimental phase diagram showing that the $\gamma$-loop extended to 11.9 at. \% Cr. Of course, the shape of the $\gamma$-loop predicted by the magnetic cluster expansion was different from the shape observed experimentally, indicating a need for an improvement of the model. In particular, Korzhavyi et al. [114] pointed out that the magnetic cluster expansion, Eq. (10), is based on the structure inversion method (SIM). The effective interactions in an alloy entering Eq. (10) were derived from the calculated enthalpies of formation for a set of Fe-Cr superstructures, which were all considered in the ferromagnetic state. Thus, the approach missed the dependence of the interactions on the magnetic state, volume, as well as on concentration, which strongly influenced the interactions $[113,114,115,120]$, changing them so dramatically that a transformation of the concentration-, volume- and magnetic-state-dependent interactions to the usual concentration-, volume- and magnetic-state-independent forms, as assumed in SIM, seemed to be a formidable and hardly achievable task. As a matter of fact, similar shortcomings of the scheme are relevant to the recent application of the magnetic cluster expansion to simulations of $f c c$ Fe-Ni alloys [121]. Thus, more work can be expected on simulations of Fe-Cr system, as well as on Fe-Cr based alloys containing other alloying elements, like Ni, Mn, and Mo.

Fe-Mn alloys represent another system where simulations of magnetic disorder have made impact [122]. It has come into focus of intense research within the last 5 years due to technological importance of high-manganese steels (15 to 35 at.-\%). The steels exhibit superior ductility and extraordinary strengthening behaviour during plastic deformation due to different metal-physical deformation mechanisms, providing, e.g. a great potential for structural components in automotive engineering. The microstructure parameter that attracted the highest interest in this respect was the stacking fault energy (SFE). It determines the deformation mechanisms, the transformation induced plasticity (TRIP) or the twinning-induced plasticity (TWIP). In Fe-Mn alloys, the SFE is believed to depend strongly on the chemical composition. Unfortunately, calculation of the SFE in magnetic alloys, especially including the finite temperature effects still represents a significant challenge for the $a b$ initio theory, because of difficulties with the proper description of the atomic-scale structure of the SF and a need for the accurate treatment of lattice vibrations and thermally induced magnetic excitations [123]. On the other hand, SFE can be estimated from the structural energy difference between $f c c$ and $h c p$ alloys. In the simplest approximation, one just calculates this difference. In a somewhat more advanced approach, SFE $\gamma$ is evaluated using the axial next-nearest-neighbor Ising (ANNNI) model [106] as $\gamma(T)=G_{\text {hcp }}(T$ ) $+2 G_{\mathrm{dhcp}}(T)-3 G_{\mathrm{fcc}}(T)$, which still brings the problem to an evaluation of the Gibbs free energies of defect-free solid solutions with hcp, double-hcp ( $d h c p$ ) and fcc crystal structures. 
Gebhardt et al.[124] used the former model and studied the lattice stability of random Fe-Mn alloys. Considering the $f c c$ phase, which has $T_{N}$ above room temperature as magnetically ordered (antiferromagnetic), while simulating the $h c p$ alloys with $T_{N}$ below room temperature within DLM-CPA Gebhardt et al. observed a crossover of the lattice stability with increasing Mn concentration, from $h c p$ to $f c c$, at approximately 24 at.\% $\mathrm{Mn}$, in good agreement with thermodynamic calculations ( 26 at.\% Mn). On the contrary, when both phases were considered as paramagnetic, the $h c p$ alloys were found to be more stable with respect to the $f c c$ alloys at all considered compositions, 15-40 at.\% Mn. Interestingly, the increase of the Mn content was found to stabilized the hcp phase further, while thermodynamic calculations displayed a contrary trend. Gebhardt et al. explained the disagreement by a complete neglect of magnetic effects in the thermodynamic modeling.

Reyes-Huamantinco et al. [123] used the ANNNI model for the estimation of the SFE in Fe-22.5 at.\% Mn alloy. Moreover, beyond the total energy contributions calculated at zero temperatures, Reyes-Huamantinco et al. included other contributions to the free energy, e.g. the vibrational and magnetic contributions in Eq (1). Of course, very serious approximations had to be involved. In particular, magnetic thermal excitations have been considered by modeling longitudinal spin fluctuations using Hamiltonian (11), followed by the use of the calculated average magnitudes of the DLM magnetic moments $m_{p}$ in an expression for magnetic entropy $S_{m a g}=k B \ln \left(m_{p}+1\right)$. The effects of lattice vibrations were treated within the Debye-Grüneisen model. The limitations of these models showed up, for instance, in a strongly overestimated value of the thermal expansion coefficient and in certain quantitative discrepancy of the SFE with the experimental data. Despite this, the temperature dependence of the SFE was reproduced quite accurately in Ref. [123]. Thus, calculations by Reyes-Huamantinco et al. clearly demonstrated the need to considering the interplay between the thermally induced magnetic excitations and the lattice vibrations for accurate treatment of the phase stability in magnetic alloys.

In fact, we would like to make another warning on ab initio simulations of Fe-Mn alloys, which most probably should be considered in all other Fe-based systems. Ekholm and Abrikosov [125] considered magnetically ordered $f c c$ alloys, and demonstrated that the two most frequently used GGA parameterizations not only failed to reproduce the equilibrium lattice constant of FeMn alloys, and consequently the magnetic properties, but also internally yield qualitatively different results. This underlined the limitations of conventional local (LSDA) and semi-local (GGA) approximations for magnetic materials containing transition metals, a conclusion which is in line with the one drawn by Dick et al. [126] in the studies of cementite. Also, the observation is consistent with the importance of correlation effects for the description of ground state properties of hcp Fe, emphasized recently in Refs. [93, 95] on the basis of the DMFT calculations. 
However, Ekholm and Abrikosov also demonstrated that for practical studies of Fe-Mn alloys, a set of approximations used by Gebhardt et al. [124] turned out to be internally consistent, and gave the equilibrium lattice constant and magnetic properties in good agreement with the experiment in the whole range of alloy compositions. Supported by very good agreement with experiment, this scheme allowed for important follow up studies, e.g. of the influence of additions of $\mathrm{Al}$ and $\mathrm{Si}$ on the lattice stability of $f c c$ and $h c p$ Fe-Mn random alloys [127], as well as elastic properties of Fe-Mn-based alloys [128], discussed in Sec. 7.5

\subsection{Formation energies of point defect in Fe}

Finite temperature properties of defects play an important part in determining high-temperature deformation modes of structural materials, such as iron alloys and steels. The impurities and alloying elements play a decisive role in the microstructure formation. For example, a precipitation of carbides and nitrides of $\mathrm{V}$ and $\mathrm{Nb}$ increases strength of the steels and prevents the austenite grain growth during hot rolling. Clusters of interstitial $\mathrm{Cr}$ atoms have been identified as the primary reason for radiation induced swelling of RPV steels in modern nuclear reactors. High-manganese steels (15-35 at.\%) exhibit superior mechanical properties, which are ultimately related to the energies of stacking faults, as has been discussed in Sec. 7.2. In turn, DFT calculations show substantial variations of magnitudes of magnetic moments in the strongly distorted core regions of defect structures, at surfaces and interfaces [13]. A recent review of this issue can be found in [129]. Unfortunately, in most cases the defects in magnetic materials are still simulated using approximations of the complete magnetic order, or treating the paramagnetic state as non-magnetic. Thus, simulations of defects in magnetic materials above the magnetic transition temperature represent an important task for the field.

In particular, Gorbatov et al. [130] carried out a systematic study of solution energies for $3 d$ impurities in paramagnetic $b c c$ Fe using the DLM approach similar to the one described in Sec. 5.1. The solution energies were found to vary regularly depending on the position of the element in the Periodic Table, underlying the fact that the main factor that determined the solubility of the $3 d$ elements was their electronic structure. Interestingly, quite strong dependence of impurities characteristics on the magnetic state of the matrix was observed, especially with respect to the magnetic moments on the impurities. Moreover, the impurity solution energies differed significantly between ferromagnetic and DLM calculations for $\mathrm{V}, \mathrm{Cr}$, $\mathrm{Co}$ and $\mathrm{Cu}$, underlying the necessity of a proper treatment of the magnetic state of the matrix in these types of studies. Unfortunately, the effects of local lattice relaxations in the paramagnetic state were neglected in this study, because the use of spherical approximation for one-electron potential in the DLM calculations. 
Vacancy formation energies in paramagnetic bcc Fe have been calculated by Ruban and Razumovskiy [75] using the SWM formalism. Very good agreement with the experiment has been obtain in calculations that have included the effect of magnetic short-rang order, $1.90 \mathrm{eV}$ (theory, $1200 \mathrm{~K}$ ) vs $1.79 \mathrm{eV}$ (experiment), though the ratio between the vacancy formation energies in the ferromagnetic and paramagnetic bcc Fe was somewhat overestimated in theory. Later, Ding et al. [131] demonstrated that improving the accuracy of the calculations, the results for the difference mentioned above could be improved. Moreover, using the SWM Ding et al. have computed vacancy formation energy differences between ferromagnetic and paramagnetic states also for $\mathrm{Mn}$ and $\mathrm{Co}$ and investigated the trend in the series Mn-Fe-Co for the bcc and fcc crystal structures. There results have shown a certain crystal structure effect (the energy differences between ferromagnetic and paramagnetic states have been less pronounced in the latter structure), but the largest effect have been shown to come due to the transition from "weak" ferromagnets (Fe and Mn with a strong effect) to the "strong" ferromagnet Co, where the effect have been almost absent.

Moreover, in addition to the vacancy formation energies, Ding et al. [131] calculated vacancy migration energies for $b c c$ Fe and $f c c$ Co, both in the ferromagnetic and the paramagnetic states, as well as diffusion coefficients. Calculated results for the latter have accurately reproduced measured values for $T / T_{C}$ ranging from 0.7 to 1.1, including the anomaly in the Arrhenius plot for Fe near $T_{C}$. Note, however, that the influence of the magnetic state on the relaxation energy around the defect, as well as on the diffusion prefactor have not been included in calculations by Ding et al., who extracted these values from first-principles simulations in the ferromagnetic state.

Ponomareva et al. [76,132] have carried out a study of substitutional (V, Nb) and interstitial (C, $\mathrm{N}$ ) impurities in paramagnetic $f c c$ Fe using SQS-MSM method (Sec. 5.2). A summary of results obtained in these works is given in Table I. Calculated solution enthalpies for $\mathrm{C}, \mathrm{N}, \mathrm{V}$, and $\mathrm{Nb}$ impurities qualitatively agree with known experimental trends. In fact, SQS-MSM approach gives estimations of solution enthalpies with accuracy similar to what is expected for firstprinciples calculations in magnetically ordered and non-magnetic materials, although, as the calculated and measured solution enthalpies are small relative deviations between them are significant in the cases of $\mathrm{C}$ and $\mathrm{N}$. Nevertheless, solubility products for corresponding carbides and nitrides in the paramagnetic state calculated in [76] have been found in good quantitative agreement with available experimental data. Similarly, C-C impurity interactions, which have been calculated in [132] have been found in good agreement with semi-empirical estimations from the literature (Table I). 
Table I. Values of solution enthalpy ( $\left\langle H_{\text {sol }}^{I}\right\rangle$, in $\mathrm{eV}$ ) for C, N, V, and Nb impurities, as well as C-C impurity interactions $\left(\left\langle E_{C C}^{\mathrm{int}}(i)\right\rangle\right.$, in $\left.\mathrm{eV}\right)$ as a function of coordination shell $i$ in paramagnetic fcc iron.

\begin{tabular}{|c|c|c|c|c|c|c|c|c|}
\hline & $\left\langle H_{\text {sol }}^{C}\right\rangle$ & $\left\langle H_{\text {sol }}^{N}\right\rangle$ & $\left\langle H_{\text {sol }}^{V}\right\rangle$ & $\left\langle H_{\text {sol }}^{N b}\right\rangle$ & $\left\langle E_{C C}^{\mathrm{int}}(1)\right\rangle$ & $\left\langle E_{C C}^{\text {int }}(2)\right\rangle$ & $\left\langle E_{C C}^{\mathrm{int}}(3)\right\rangle$ & $\left\langle E_{C C}^{\mathrm{int}}(4)\right\rangle$ \\
\hline $\begin{array}{l}\text { MSM- } \\
\text { SQS }\end{array}$ & $0.20^{\mathrm{a}}$ & $-0.39^{\mathrm{a}}$ & $-0.24^{\mathrm{a}}$ & $-0.36^{a}$ & $0.18^{\mathrm{b}}$ & $0.17^{\mathrm{b}}$ & $-0.004^{b}$ & $0.004^{\mathrm{b}}$ \\
\hline $\begin{array}{l}\text { Other } \\
\text { works }\end{array}$ & $\begin{array}{l}0.36- \\
0.43^{\mathrm{c}}\end{array}$ & $-0.18^{c}$ & & & $\begin{array}{l}0.086- \\
0.15^{d}\end{array}$ & $\begin{array}{l}0.17- \\
0.40^{\mathrm{d}}\end{array}$ & $-0.042^{\mathrm{d}}$ & $0.04^{\mathrm{d}}$ \\
\hline
\end{tabular}

${ }^{\mathrm{a}}$ Ref. [76]; ${ }^{\mathrm{b}}$ Ref. [132]; ${ }^{\mathrm{c}}$ Experimental estimations from [133]; ${ }^{\mathrm{d}}$ Semiempirical estimations from [134].

\subsection{First-principles simulations of the paramagnetic state of transition metal compounds: the metal-to-insulator transition}

Properties of transition-metal compounds are of central importance to condensed matter theory because of their fundamental scientific interest and the importance for technological applications. Let us take iron oxides as an example. They are products of iron corrosion in air, one of the most detrimental processes from the technological perspective. In addition, the iron oxide is one of the fundamental components in the Earth's lower mantle. Finally, FeO has been in focus of experimental and theoretical studies of the metal-to-insulator transition (MIT), one of the central issues of the condensed matter physics. Transition metal oxides, carbides and nitrides are used in many applications, ranging from electronics to hard coatings of cutting tools. In many cases, the magnetic transition temperatures of transition metal compounds are quite low. For instance, $\mathrm{V}_{2} \mathrm{O}_{3}, \mathrm{MnO}$, FeO, the prototype materials for the studies of the MIT [8, 70,135,136], all have $T_{N}$ below the room temperature. On the other hand, magnetic properties of the transition metal compounds above the magnetic transition temperature are highly important for the description of their other physical properties, like mechanical and thermodynamic properties [17].

One of the main issues that one should have in mind dealing with transition metal compounds is that for many of them the local and semi-local approximations within the DFT fail to reproduce the correct electronic ground state, predicting them to be metals rather than insulators. Thus, more advanced computational schemes, like the LDA+DMFT (Sec. 3) are often employed. In fact, the LDA+DMFT is believed to be the best available method for the investigation of real 
systems close to a Mott-Hubbard MIT [70]. It was applied with success for the studies of this problem in classical systems, $\mathrm{V}_{2} \mathrm{O}_{3}$ [70], $\mathrm{MnO}$ [8], $\mathrm{FeO}$ [135,136], $\mathrm{Fe}_{2} \mathrm{O}_{3}$ [137], as well as in other materials, e.g. rare-earth-element nickelates [71]. The nickelates attracted significant attention in recent years. Their insulating state was characterized by a two-sublattice symmetry breaking.

At the same time, an application of the LDA+DMFT approach is still a challenge, because of numerical complexity, as well as some fundamental challenges (see Sec. 3 for the discussion). For instance, the former forced Held et al. [70] to approximate the effect of $\mathrm{Cr}$ doping of $\mathrm{V}_{2} \mathrm{O}_{3}$ by carrying out calculations for stoichiometric $\mathrm{V}_{2} \mathrm{O}_{3}$ compound with an expanded lattice parameter. While well justified for the purpose of study presented in Ref. [70], such simplifications could be too strong for other tasks. Another interesting example is represented by the LDA+DMFT studies of FeO carried out by Shorikov et al. [135] and by Ohta et al. [136]. Though the both groups reproduced the MIT, they arrived at quite different conclusions with respect to its details. In the former work, the metallization was found at $60 \mathrm{GPa}$ already at room temperature. Moreover, Shorikov et al. found that in $\mathrm{FeO}$ average value of magnetic moment was nearly the same in the insulating phase at ambient pressure and in the metallic phase at high pressure. On the contrary, in calculations by Ohta et al. [136] FeO metallized at around 70 GPa, but at high temperature of $1900 \mathrm{~K}$, and the observed metallization was related to spin crossover, similar to $\mathrm{MnO}$ [8] and $\mathrm{Fe}_{2} \mathrm{O}_{3}$ [137] where metal-to-insulator transition was also accompanied by the high-spin to low-spin transition. Ohta et al. pointed out to several differences in their calculations and in the calculations by Shorikov et al., e.g. that the calculations in Ref. [135] were restricted only to Fe $3 d$ orbitals, and they were not charge self-consistent. This discrepancy underlines that the use of the LDA+DMFT approach is still not straightforward.

The LDA+U method is significantly less demanding numerically than the LDA+DMFT. However, while it can be easily used to describe the magnetically ordered phases, it is generally believed that the correlated paramagnetic phases are beyond the scope of the LDA+U approach since the Coulomb interaction is treated within Hartree-Fock [70,71]. However, Alling et al. [72] suggested to using $\mathrm{LDA}+\mathrm{U}$ calculations in combination with supercell approaches for the treatment of magnetic disorder, magnetic SQS or MSM methods (Sec. 5.2). For instance, using this combination, Alling et al. successfully reproduced the electronic structure of cubic B1 phase of CrN, a well-known example of correlated paramagnetic material (see Fig. 10). A combination of the LDA+U and the DLM-MD (Sec. 6.1) allowed Alling et al.to approach, with success, such complex problems, as a description of substitutional disordered in correlated paramagnetic materials at finite temperatures [77]. 


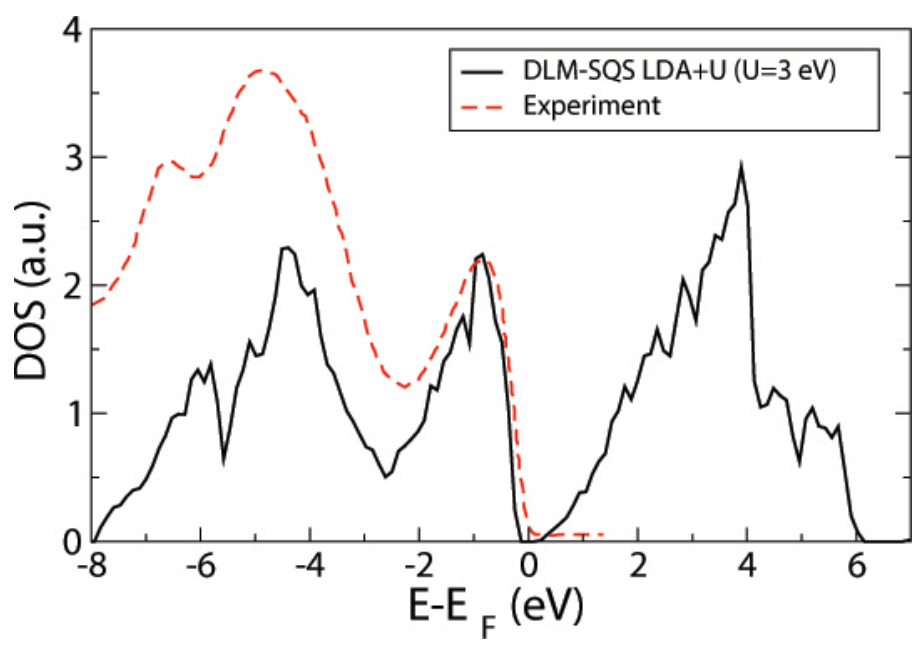

Figure 10. Valence-band electronic density of states (solid line) of the paramagnetic cubic B1 phase of CrN calculated in Ref. [60] using LDA+ $U$ approximation (with $U=3 \mathrm{eV}$ ) and a description of magnetic disordered by means of the supercell approach. The experimental ultraviolet photoemission spectroscopy measurement from [138] is shown by a dashed line.

Of course, for large groups of transition metal compounds LSDA or GGA calculations are sufficient, and they are used with considerable success. Enormous interest is generated by great importance of this class of systems, and a full survey of recent literature in this field would be too extensive. We therefore restricted ourselves by the consideration of methodological challenges to dealing with the paramagnetic state of magnetic transition metal compounds.

\subsection{Simulations of elastic properties.}

Performance of steels is based on an impressive variety of competing mechanisms on the microscopic/atomic scale, and includes dislocation gliding, solid solution hardening, mechanical twinning or structural phase transformations [109]. Among their most important properties are the elastic properties, which determine mechanical response of the system. Moreover, it has been demonstrated that the elastic and the plastic processes simultaneously involved in the deformation may be closely related. For example, there exists a significant correlation between the product of the macroscopic parameters of localized plastic flow auto-waves in deforming alloys, their length and propagation rate (Fig. 11) and the product of the microscopic (lattice) parameters of these materials, the spacing between close-packed planes of the lattice and the rate of transverse elastic waves [140]. A possibility to predict elastic properties of magnetic materials, e.g. in the paramagnetic state is therefore highly requested. 


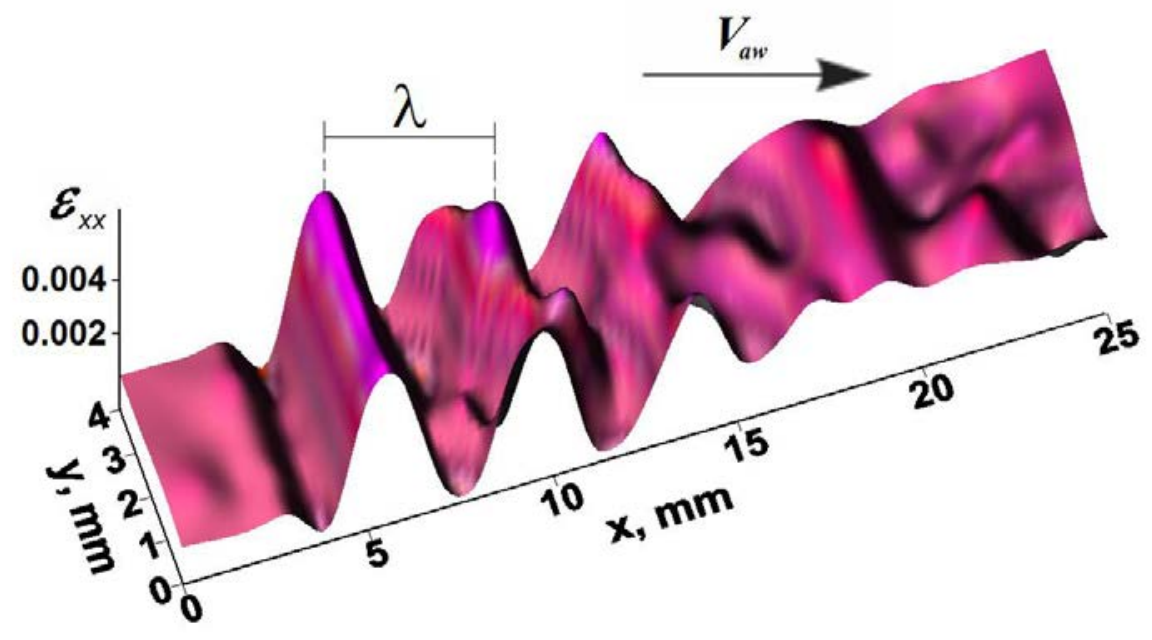

Figure 11. Example of localized plastic flow autowave generated at the linear work hardening stage in the single crystal of fcc Fe $-18 \% \mathrm{Cr}-12 \% \mathrm{Ni}-2 \%$ Mo alloy oriented along [001] direction; $\varepsilon_{x x}$ - local elongation; $x$ and $y$-specimen length and width, respectively; $\lambda$ - nucleus spacing (autowave length); $V_{a w}$ - autowave propagation rate. The kinetics of macro-localization pattern evolution was investigated using time evolution of local nuclei’s positions [139].

Starting with pure Fe, Leonov et al. [44] computed elastic properties of paramagnetic bcc and fcc phases of this metal using the result for the phonon dispersions, calculated within the LDA+DMFT approach at reduced temperatures $1.2 T_{C}$ and $1.4 T_{C}$, respectively. Zhang et al. Ref. [90] carried out similar calculations within DLM-CPA picture using conventional DFT calculations. Ruban and Razumovskiy calculated elastic properties of paramagnetic bcc Fe using both, DLM-CPA and the SWM [75]. A comparison of all three data sets with experiment can be found in Ref. [75]. The average deviation between calculated and experimental results, independently on the methodology used, is around 30\%. But in some cases, especially if corresponding elastic moduli are small, like $C^{\prime}$ in bcc Fe (13.3 GPa at $1173 \mathrm{~K}$ ), the spread between different theoretical results is significant, ranging from -5 GPa in calculations within the SWM without magnetic short-range order to $36 \mathrm{GPa}$ in LDA+DMFT calculations. In addition, the elastic moduli $C_{11}$ calculated within LDA+DMFT approach for the paramagnetic fcc Fe (210 GPa) differs significantly from experiment (154 GPa). Note that the elastic moduli $C^{\prime}$ and $C_{11}$ are directly involved in the structural transformation path (the so-called Bain path) from bcc to $f c c$, and therefore are critical to understanding mechanical failure of ferritic steels at high $T$ [86].

Several comments should be made at this point. First, magnetic short-range order present in the paramagnetic bcc Fe at temperatures of interest may significantly affect the results of the 
calculations. For instance, in calculations by Ruban and Razumovskiy [75] C' has increased from -5 GPa to $10 \mathrm{GPa}$ when the magnetic short-range order have been included. Perhaps almost perfect agreement of the calculated $C$ ' with experiment has been fortuitous. However, the most important improvement has been at a qualitative level: the bcc phase of Fe has become mechanically stable.

Next, the main way how the temperature has been incorporated in the above mentioned calculations has consisted of approximate (that is, excluding the implicit effect of lattice vibrations) $a b$ initio [44] or empirical [75, 90] incorporation of the thermal expansion of the lattice. While it is believed to be the most important contribution to the temperature dependence of the elastic moduli, it would be very interesting to investigate directly the full effect of lattice dynamics. Such an opportunity is offered by the DLM-MD approach (Sec. 6.1). Figure 12 shows equations of state of orthorhombic antiferromagnetic phase of $\mathrm{CrN}$, calculated with conventional $a b$ inito MD, and cubic (B1) paramagnetic phases of CrN, calculated with the DLM-MD. The accuracy of the calculations for the paramagnetic phase is comparable to that of conventional $a b$ inito MD simulations for magnetically ordered materials. Both results are in excellent agreement with experiment.

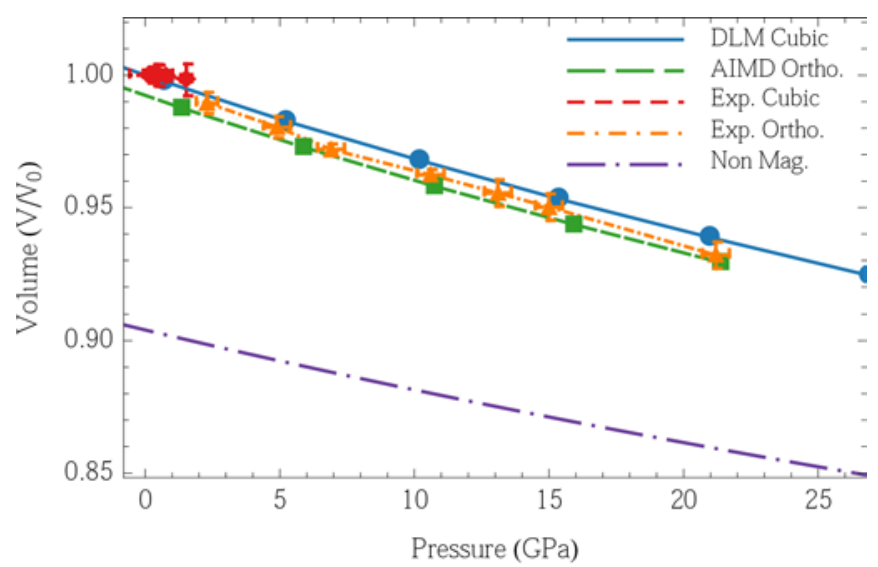

Figure 12. (Color online) Equations of state of orthorhombic antiferromagnetic phase of $\mathrm{CrN}$, calculated with conventional $a b$ inito MD (green squraes), and cubic (B1) paramagnetic phases of CrN, calculated with DLM-MD (blue circles). Experimental data for the orthorhombic (orange triangles up) and cubic (red triangles down) phases are from Ref. [16]. The strong effect on the equation of state due to the neglect of magnetism is demonstrated by non-magnetic calculations shown with long dashed - dotted line. 

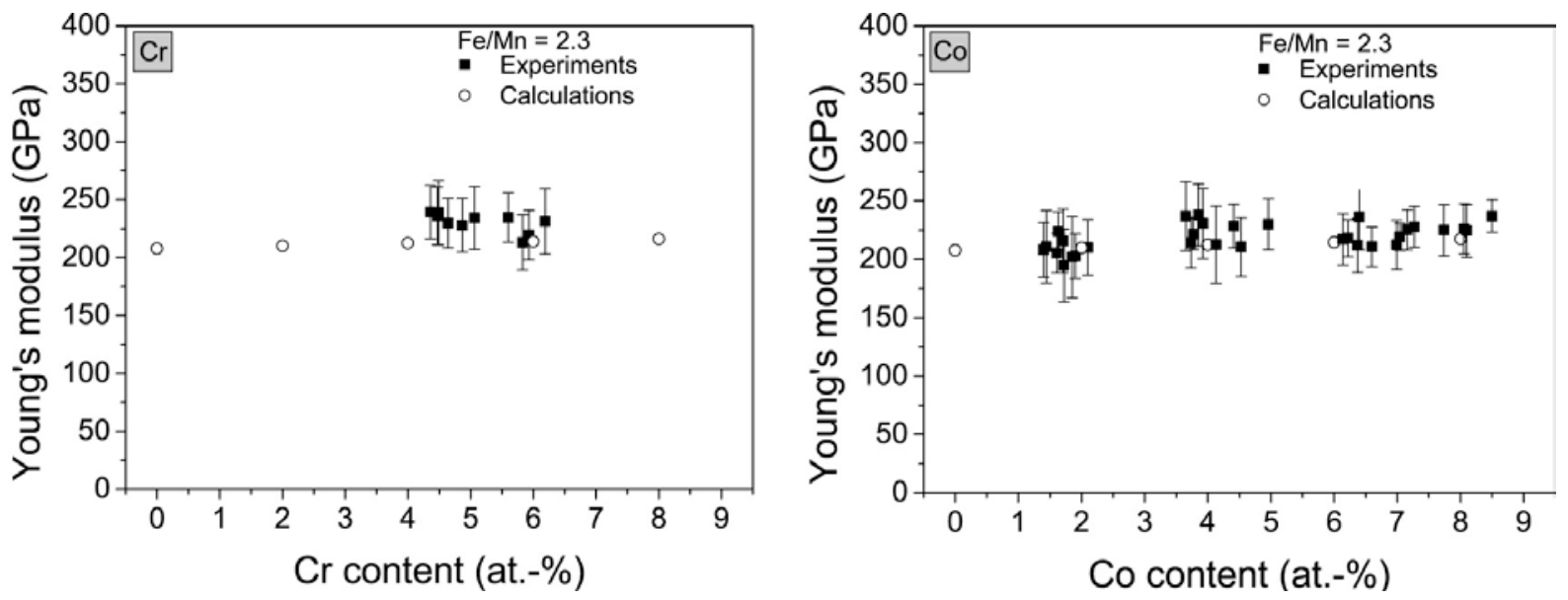

Figure 13. Calculated (open circles) and experimentally determined (filled rectangles) Young's modulus values for fcc Fe-Mn-Cr and Fe-Mn-Co with a Fe=Mn ratio of 2.3. From [128]. (C) IOP Publishing. Reproduced with permission. All rights reserved.

On the other hand, calculations of elastic constants of paramagnetic phases of Fe at very high temperatures represent extreme challenge for modern theory. In many cases, one deals with materials with significantly lower magnetic transition temperatures. Usually their elastic properties are better reproduced in static DFT calculations that model the magnetic disorder in one or another way. In particular, DLM-CPA approach (Sec. 5.1) has been highly successful. Its recent applications include calculations of elastic moduli in Fe-Cr [141], Fe-Ga [142], $\mathrm{Ni}_{3} \mathrm{Fe}$ [143], and multicomponent Fe-Mn alloys [128]. As an example, we show in Fig. 13 excellent agreement between calculated (within DLM-CPA) and experimentally determined room temperature Young's modulus values for $f c c \mathrm{Fe}-\mathrm{Mn}-\mathrm{Cr}$ and $\mathrm{Fe}-\mathrm{Mn}-\mathrm{Co}$ alloys, reported in Ref. [128].

Further, Vitos et al. used the DLM-CPA calculations for guiding a design of austenitic stainless steels with the aim to combine high mechanical characteristics with good resistance against localized corrosion [65]. Cubic elastic moduli $C_{11}, C_{12}$ and $C_{44}$ of $f c c$ Fe-Cr-Ni alloys were calculated from first principles in the whole composition range. They were found to be in good agreement ( $\sim \%$ ) with available experiment. From the values of the single-crystal elastic moduli, a database of the polycrystalline shear modulus $G$ and bulk modulus $B$ were established, and it was shown that an optimal combination of $G$ and $B$ should be achieved in alloys within the compositional range of commercial stainless steels. In particular, Vitos et al. predicted that $\mathrm{Fe}_{58} \mathrm{Cr}_{18} \mathrm{Ni}_{24}$ alloys possess an intermediate hardness combined with improved ductility and excellent corrosion resistance. Then a study of additional alloying elements on elastic properties, lattice parameters and lattice stability of Fe-Cr-Ni alloys was carried out. As a result, 
$\mathrm{Fe}_{58} \mathrm{Cr}_{18} \mathrm{Ni}_{24}$ austenitic alloys containing a few per cent Os or Ir were proposed for practical applications.

Here we note that the cost optimization is seldom present in theoretical considerations. However, in a recent review by Sandström and Korzhavyi [144] the use of materials optimization techniques combined with first-principles calculations of the elastic moduli to develop new materials that are systematically adapted to specific components has been discussed. In particular, cost, weight, and environmental impact minimization can be included in the design criteria. In addition, this review contains excellent collection of literature on $a b$ initio calculations of elastic constants, e.g. in the paramagnetic state of magnetic alloys.

\section{Conclusions and outlook}

The effects of finite temperature magnetic excitations in magnetic materials on their structural and elastic properties are still not well understood. Because of the complexity of the problem, they are seldom included in first principles calculations. Their neglect is often accompanied by an argument that they should be small, and that these are the second-order effects in simulations of phase stability and mechanical behavior. Moreover, paramagnetic phases of magnetic materials are still modeled as non-magnetic and many researchers do not distinguish between the two terms. We reaffirm that the non-magnetic and paramagnetic state should always be distinguished, as argued by many over the years. Furthermore, we provide an overview of several theoretical techniques available at present to address such states more properly including critical electronic and thermodynamic effects.

Specifically, we discuss recent developments in the field of first-principles simulations of magnetic materials in their paramagnetic state, that is above the magnetic order-disorder transition temperature. We focus on $3 d$-transition metals, their alloys and compounds. Itinerant electrons determine the magnetic properties of these systems. However, for a large class of the materials the magnetization density is well localized (Fig. 1), and each atom could be associated with a local moment parallel to the net magnetization density at the site. These local moments survive but disorder above the magnetic order-disorder transition temperature.

The magnetic disorder makes theoretical description of the system at hand highly non-trivial. Indeed, starting with the Gibbs free energy for a magnetic system in its paramagnetic state, Eq. (1) we argue that a conventional adiabatic decoupling of the terms into electronic $G_{e l}$, vibrational $G_{v i b}$, and magnetic $G_{m a g n}$ contributions may not work. This is so because of a comparable time 
scales for vibrational and magnetic excitations, as well as the effect of the latter on the potential energy surface and consequently its influence on $G_{e l}$. Thus, in principle all the terms should be treated simultaneously and at the same footing. Moreover, for the itinerant electron systems local magnetic moments become temperature dependent. Besides the thermal fluctuations, which disorder the moments, they should depend on local chemical and magnetic environment, as well as they should be influenced by the temperature induced structural disorder. In addition, a variety of competing many-body effects should be considered, such as Kondo screening and the induction of local magnetic moment by temperature.

We review theoretical tools, which allow for description of such a complex problem, focusing on their advantages and limitations. In particular, the dynamical mean-field theory gives perhaps the most consistent way to describe the paramagnetic state of a magnetic material with account of correlation effects. However, its numerical complexity, as well as conceptual challenges discussed in Sec. 3 motivate the use of alternative methods, which treat many-electron effects at more approximate levels. Based on the equivalence between a many-body interacting system with Coulomb onsite interactions and a one-electron system in fluctuating charge and spin fields, one can argue for the use of more traditional DFT-based schemes implemented in ab initio spin dynamics or its quasi- or semi-classical simplifications (Sec. 4). Moreover, employing the concept of temporarily broken ergodicity one inroduces the disordered local moment picture (Sec. 5). In the DLM the magnetically disordered state can be described as a pseudo-alloy of equal amounts of atoms with spin up and spin down orientations of their local moments, and its electronic structure and the total energy can be calculated within the tools borrowed from the alloy theory, e.g. using the coherent potential approximation or supercell approaches.

Further, we argue that contributions from lattice vibrations ought to be increasingly important when one goes from low-temperature magnetically ordered state to the high-temperature paramagnetic state. In order to couple magnetic and vibrational excitations, we introduce the concept of disordered local moment molecular dynamics (Sec 6). In addition, we present arguments that interatomic forces in the paramagnetic state may be calculated in the adiabatic limit, considering magnetic degrees of freedom as fast with respect to atomic motions and averaging over the former.

We discuss recent applications of the theories reviewed in Secs. 3-6 for calculations of thermodynamic and mechanical properties of materials in the paramagnetic state. We are mostly interested in simulations that considered effects of temperature induced magnetic disorder. In most cases, except Sec. 7.4, we are interested in simulations of thermodynamic and mechanical properties, rather than in the studies of the electronic structure and magnetic phase transitions. 
The considered examples underline the need for a proper treatment of magnetic disorder in firstprinciples simulations. They also underline challenges that remain to be solved within this field.

The following questions have to be addressed. First, an improvement on the efficiency of stateof-the-art many body techniques, like the LDA+DMFT method is highly desirable, as well as a solution of its fundamental problems, related to the presence of model parameters, difficulty to calculate interatomic forces, and the mean-field nature of the approach. Second, we foresee increased activities on combining molecular and spin dynamics into the same framework. Such combination will allow one to treat thermally induced magnetic and vibrational excitations on the same footing. Third, it will be very important to develop a consistent first-principles framework for the treatment of magnetic entropy term, which at present is most often approximated by a mean-field expression adopted for it in the thermodynamic modeling community [28]. Next, the limitations of conventional local (LSDA) and semi-local (GGA) approximations for magnetic materials containing transition metals, discussed e.g. in Sec. 7.2 would have to be addressed by the community involved in the density functionals development. Unfortunately, at present, magnetic systems are not considered as a primary task for these works. It is also clear that more work needs to be done on improving theoretical models for transition metals, like $\mathrm{Cr}$ and $\mathrm{Ni}$, which are more itinerant than $\mathrm{Fe}$, and on more consistent way to including the effects of longitudinal spin fluctuations. At last, but not least, more simulations have to be done on magnetic materials using the existing tools, especially newly developed methods to verify their reliability, or otherwise. Development of consistent theory of magneto-structural coupling will certainly give enormous new possibility for the knowledge-based design of magnetic materials.

\section{Acknowledgements}

This work was supported by the Grant of the Ministry of Education and Science of the Russian Federation (Grant No. 14.Y26.31.0005). A support provided by Tomsk State University Academic D.I. Mendeleev Fund Program (project No. 8.1.18.2015), the Swedish Foundation for Strategic Research (SSF) program SRL Grant No. 10-0026, the Swedish Research Council (VR) Grants No. 621-2011-4426 and 621-2011-4417, and the Program of Fundamental Research of State Academies of Sciences for the period 2013-2016 yrs. (No. III.23.1.2) are gratefully acknowledged. Calculations were performed at the Swedish National Infrastructure for Computing (SNIC), at computer cluster "Cherry" at NUST 'MISIS' and at the Joint Supercomputer Center of the Russian Academy of Sciences (Moscow). 


\section{REFERENCES}

[1] The Materials Genome Initiative for Global Competitiveness; 2011, http://www.whitehouse.gov/sites/default/files/microsites/ostp/materials_genome_initiative$\underline{\text { final.pdf }}$

[2] See, for example, a series Ferromagnetic Materials, edited by E. P. Wohlfarth and K. H. J. Buschow. Amsterdam: North-Holland; 1980-1993, Vols. 1-7.

[3] Yu XZ, Onose Y, Kanazawa N, Park JH, Han JH, Matsui Y, Nagaosa N and Tokura Y. Real-space observation of a two-dimensional skyrmion crystal. Nature 2010; 465: 901-904. doi:10.1038/nature09124

[4] Hohenberg P and Kohn W. Inhomogeneous Electron Gas. Phys. Rev. 1964; 136 : B864. Kohn W. and Sham LJ. Self-Consistent Equations Including Exchange and Correlation Effects. Phys Rev 1965; 140: A1133.

[5] Martin RM. Electronic structure. Basic Theory and Practical Methods. Cambridge : Cambridge University Press; 2004.

[6] Wang Y, Stocks GM, Nicholson DMC, Shelton WA, Antropov VP, Harmon BN . Noncollinear magnetic structure in Ni0.35Fe0.65. J Appl Phys 1997; 81: 3873-3875.

[7] Schilfgaarde M, Abrikosov IA and Johansson B. Origin of the Invar effect in iron-nickel alloys. Nature 1999; 400: 46-49. doi:10.1038/21848

[8] Kunes J, Lukoyanov AV, Anisimov VI, Scalettar RT and Pickett WE. Magnetic Moment Collapse-Driven Mott Transition in MnO. Nature Mater 2008; 7:198-202. doi:10.1038/nmat2115

[9] Antropov VP, . Katsnelson MI, Schilfgaarde M, Harmon BN. AbInitio Spin Dynamics in Magnets. Phys Rev Lett 1995; 75: 729-732. DOI: http://dx.doi.org/10.1103/PhysRevLett.75.729 [10] Antropov VP, Katsnelson MI, Harmon BN, Schilfgaarde M, Kusnezov D. Spin dynamics in magnets: Equation of motion and finite temperature effects.

Phys Rev B 1996; 54: 1019-1035 (1996). DOI: http://dx.doi.org/10.1103/PhysRevB.54.1019

[11] Hellsvik J, Skubic B, Nordström L, Sanyal B, Eriksson O, Nordblad P, Svedlindh P. Dynamics of diluted magnetic semiconductors from atomistic spin-dynamics simulations: Mndoped GaAs. Phys Rev B 2008; 78:144419. DOI: http://dx.doi.org/10.1103/PhysRevB.78.144419 
[12] Skubic B, Hellsvik J, Nordström L and Eriksson O. A method for atomistic spin dynamics simulations: implementation and examples. J Phys.: Condens. Matter 2008; 20: 315203. doi:10.1088/0953-8984/20/31/315203

[13] Ma PW and Dudarev SL. Longitudinal magnetic fluctuations in Langevin spin dynamics. Phys Rev B 2012; 86: 054416.

DOI: http://dx.doi.org/10.1103/PhysRevB.86.054416

[14] Staunton JB, Johnson DD , Gyorffy BL. Interaction between magnetic and compositional order in Ni-rich $\mathrm{Ni}_{\mathrm{c}} \mathrm{Fe}_{1-\mathrm{c}}$ alloys. J Appl Phys 1987; 61: 3693-6.

[15] J.B. Staunton JB, D.D. Johnson DD, and F.J. Pinski FJ. Theory of Compositional Correlations in Magnetic Alloys: Interpretation of a Diffuse Neutron Scattering Experiment on an IronVanadium Single Crystal. Phys Rev Lett 1990; 65:1259-62.

DOI: http://dx.doi.org/10.1103/PhysRevLett.65.1259

[16] Rivadulla F, Bañobre-López M, Quintela CX, Piñeiro A, Pardo A, Baldomir D, et al. Reduction of the bulk modulus at high pressure in CrN. Nature Mater 2009; 8: 947-951.

[17] Alling B, Marten T, Abrikosov IA. Questionable collapse of the bulk modulus in CrN. Nature Materials 2010; 9: 283-284. Alling B, Marten T, Abrikosov IA. Effect of magnetic disorder and strong electron correlations on the thermodynamics of CrN. Phys Rev B 2010; 82:184430.

[18] Crisan V, Entel P, Ebert H, Akai H, Johnson DD, Staunton JB. Magnetochemical origin for Invar anomalies in iron-nickel alloys. Phys Rev B 2002; 66:014416.

DOI: http://dx.doi.org/10.1103/PhysRevB.66.014416

[19] Smirnov AV, Shelton WA, Johnson DD. Importance of thermal disorder on the properties of alloys: Origin of paramagnetism and structural anomalies in bcc-based Fe1-xAlx. Phys Rev B 2005;71: 064408. DOI: http://dx.doi.org/10.1103/PhysRevB.71.064408

[20] Ekholm M, Zapolsky H, Ruban AV, Vernyhora I, Ledue D, Abrikosov IA. Influence of the Magnetic State on the Chemical Order-Disorder Transition Temperature in Fe-Ni Permalloy. Phys Rev Lett 2010; 105:167208. DOI: http://dx.doi.org/10.1103/PhysRevLett.105.167208

[21] Heisenberg W. Zur Theorie des Ferromagnetismus. Z Phys 1928; 49: 619-636.

[22] Stoner EC. Collective electron specific heat and spin paramagnetism in metals. Proc Roy Soc A 1936; 154: 656-678. Stoner EC. Collective electron ferromagnetism. Proc Roy Soc A 1938; $165: 372$.

[23] Rhodes P, Wohlfarth EP. The Effective Curie-Weiss Constant of Ferromagnetic Metals and Alloys. Proc R. Soc. Lond. A 1963; 273: 247-258. 
[24] James P, Eriksson O, Johansson B, Abrikosov IA. Calculated magnetic properties of binary alloys between Fe, Co, Ni, and Cu. Phys Rev B 1999; 59: 419.

DOI: http://dx.doi.org/10.1103/PhysRevB.59.419

[25] Gyorffy BL, Pindor AJ, Staunton J, Stocks GM, Winter H. A first-principles theory of ferromagnetic phase transitions in metals. Journal of Physics F: Metal Physics 1985; 15: 13371386. doi:10.1088/0305-4608/15/6/018

[26] Moriya T. Recent progress in the theory of itinerant electron magnetism. J Magn Magn Mater 1979; 14: 1. Moriya T. Spin Fluctuations in Itinerant Electron Magnetism. Berlin: Springer-Verlag, 1985.

[27] Steneteg P, Alling B, Abrikosov IA. Equation of state of paramagnetic CrN from $a b$ initio molecular dynamics. Phys Rev B 2012; 85:144404. DOI:

http://dx.doi.org/10.1103/PhysRevB.85.144404

[28] Körmann F, Breidi AAH, Dudarev SL, Dupin N, Ghosh G, Hickel T, Korzhavyi P, et al. Lambda transitions in materials science: Recent advances in CALPHAD and first-principles modelling. Phys Status Solidi B 2014; 251: 53-80. DOI 10.1002/pssb.201350136

[29] Staunton JB, Gyorffy BL. Onsager cavity fields in itinerant-electron paramagnets. Phys Rev Lett 1992; 69:371. DOI: http://dx.doi.org/10.1103/PhysRevLett.69.371

[30] Uhl M, Kübler J. Exchange-Coupled Spin-Fluctuation Theory: Application to Fe, Co, and Ni. Phys Rev Lett 1996; 77: 334. DOI: http://dx.doi.org/10.1103/PhysRevLett.77.334

[31] Rosengaard NM, Johansson B. Finite-temperature study of itinerant ferromagnetism in Fe, Co, and Ni. Phys Rev B 1997; 55:14975. DOI: http://dx.doi.org/10.1103/PhysRevB.55.14975

[32] Ruban AV, Khmelevskyi S, Mohn P, Johansson B. Temperature-induced longitudinal spin fluctuations in Fe and Ni. Phys Rev B 2007; 75: 054402.

DOI: http://dx.doi.org/10.1103/PhysRevB.75.054402

[33] Wysocki AL, Glasbrenner JK, Belashchenko KD. Thermodynamics of itinerant magnets in a classical spin-fluctuation model. Phys Rev B 2008; 78: 184419.

DOI: http://dx.doi.org/10.1103/PhysRevB.78.184419

[34] Lavrentiev MY, Nguyen-Manh D, Dudarev SL. Magnetic cluster expansion model for bccfcc transitions in Fe and Fe-Cr alloys. Phys Rev B 2010; 81:184202.

DOI: http://dx.doi.org/10.1103/PhysRevB.81.184202

[35] Zener C. Impact of magnetism upon metallurgy. Trans. of AIME 1955; 203: 619-630. 
[36] Ruban AV and Abrikosov IA. Configurational thermodynamics of alloys from first principles: effective cluster interactions. Rep Prog Phys 2008;71: 046501.

doi:10.1088/0034-4885/71/4/046501.

[37] Lichtenstein AI, Katsnelson MI. Finite-Temperature Magnetism of Transition Metals: An $a b$ initio Dynamical Mean-Field Theory. Phys Rev Lett 2010; 87:067205.

DOI: http://dx.doi.org/10.1103/PhysRevLett.87.067205

[38] For a recent review on DMFT, see Kotliar G, Savrasov SY, Haule H, Oudovenko VS, Parcollet O, Marianetti CA. Electronic structure calculations with dynamical mean-field theory. Rev Mod Phys 2006; 78:865. DOI: http://dx.doi.org/10.1103/RevModPhys.78.865

[39] Georges A, Kotliar G, Krauth W, Rozenberg MJ. Dynamical mean-field theory of strongly correlated fermion systems and the limit of infinite dimensions. Rev Mod Phys 1996; 68: 13. DOI: http://dx.doi.org/10.1103/RevModPhys.68.13

[40] Kotliar G and Vollhardt D. Strongly correlated materials: Insights from dynamical mean field theory. Physics Today 2004; 57:53-60.

[41] Anisimov VI and Lukoyanov AV. Investigation of real materials with strong electronic correlations by the LDA+DMFT method. Acta Cryst 2014; C70: 137-159.

[42] Igoshev PA, Efremov AV, Poteryaev AI, Katanin AA, and Anisimov VI. Magnetic fluctuations and effective magnetic moments in $\gamma$-iron due to electronic structure peculiarities. Phys. Rev. B 2013; 88:155120. DOI: http://dx.doi.org/10.1103/PhysRevB.88.155120

[43] Leonov I, Poteryaev AI, Anisimov VI, Vollhardt D. Magnetic fluctuations and effective magnetic moments in $\gamma$-iron due to electronic structure peculiarities. Phys Rev Lett 2011; 106:106405. DOI: http://dx.doi.org/10.1103/PhysRevB.88.155120

[44] Leonov I, Poteryaev AI, Anisimov VI, Vollhardt D. Calculated phonon spectra of paramagnetic iron at the $\alpha-\gamma$ phase transition. Phys Rev B 2012; 85: 020401(R). DOI: http://dx.doi.org/10.1103/PhysRevB.85.020401

[45] Hettler MH, Tahvildar-Zadeh AN, Jarrell M, Pruschke T, Krishnamurthy HR. Nonlocal dynamical correlations of strongly interacting electron systems. Phys Rev B 1998; 58:7475. DOI: http://dx.doi.org/10.1103/PhysRevB.58.R7475

[46] Maier T, Jarrell M, Pruschke T, Hettler MH. Quantum cluster theories. Rev Mod Phys 2005; 77:1027. DOI: http://dx.doi.org/10.1103/RevModPhys.77.1027

[47] Kotliar G, Savrasov SY, Palsson G, Biroli G. Cellular Dynamical Mean Field Approach to Strongly Correlated Systems. Phys Rev Lett 2001; 87:186401.

DOI: http://dx.doi.org/10.1103/PhysRevLett.87.186401 
[48] Rubtsov AN, Katsnelson MI, Lichtenstein AI. Dual fermion approach to nonlocal correlations in the Hubbard model. Phys Rev B 2008; 77: 033101.

DOI: http://dx.doi.org/10.1103/PhysRevB.77.033101

[49] Aryasetiawan F, Imada M, Georges A, Kotliar G, Biermann S, Lichtenstein AI.

Frequency-dependent local interactions and low-energy effective models from electronic structure calculations. Phys Rev B 2004; 70:195104.

DOI: http://dx.doi.org/10.1103/PhysRevB.70.195104

[50] Miyake T, Aryasetiawan F. Screened Coulomb interaction in the maximally localized Wannier basis. Phys Rev B 2008; 77:085122.

DOI: http://dx.doi.org/10.1103/PhysRevB.77.085122

[51] Leonov I, Anisimov VI, Vollhardt D. First-Principles Calculation of Atomic Forces and Structural Distortions in Strongly Correlated Materials. Phys Rev Lett 2014; 112:146401. DOI: http://dx.doi.org/10.1103/PhysRevLett.112.146401

[52] Liechtenstein AI, Katsnelson MI, Gubanov VA. Exchange interactions and spin-wave stiffness in ferromagnetic metals. J Phys F 1984; 14:L125. Liechtenstein AI, Katsnelson MI, Antropov VP, Gubanov VA. Local spin density functional approach to the theory of exchange interactions in ferromagnetic metals and alloys. J Magn Magn Mater 1987; 67: 65.

[53] Tao X, Landau DP, Schulthess TC, Stocks GM. Spin Waves in Paramagnetic bcc Iron: Spin Dynamics Simulations. Phys Rev Lett 2005; 95: 087207.

DOI: http://dx.doi.org/10.1103/PhysRevLett.95.087207

[54] Ruban AV, Shallcross S, Simak SI, Skriver HL. Atomic and magnetic configurational energetics by the generalized perturbation method. Phys Rev B 2004; 70:125115. DOI: http://dx.doi.org/10.1103/PhysRevB.70.125115

[55] Alling B, Ruban AV, Abrikosov IA. Effect of thermal expansion, electronic excitations, and disorder on the Curie temperature of Ni1-xCuxMnSb alloys. Phys Rev B 2009; 79:134417. DOI: http://dx.doi.org/10.1103/PhysRevB.79.134417

[56] Singer R, Dietermann F, Fähnle M. Spin Interactions in bcc and fcc Fe beyond the Heisenberg Model. Phys Rev Lett 2011; 107:017204.

DOI: http://dx.doi.org/10.1103/PhysRevLett.107.017204

[57] Szilva A, Costa M, Bergman A, Szunyogh L, Nordström L, Eriksson O. Interatomic Exchange Interactions for Finite-Temperature Magnetism and Nonequilibrium Spin Dynamics. Phys Rev Lett 2013; 111:127204. DOI: http://dx.doi.org/10.1103/PhysRevLett.111.127204 
[58] Lindmaa A, Lizárraga R, Holmström E, Abrikosov IA, Alling B. Exchange interactions in paramagnetic amorphous and disordered crystalline CrN-based systems. Phys Rev B 2013; 88:054414. DOI: http://dx.doi.org/10.1103/PhysRevB.88.054414

[59] Körmann F, Dick A, Hickel T, Neugebauer J. Rescaled Monte Carlo approach for magnetic systems: Ab initio thermodynamics of bcc iron. Phys Rev B 2010; 81:134425. DOI: http://dx.doi.org/10.1103/PhysRevB.81.134425

[60] Hubbard J. The magnetism of iron. Physical Review B 1979; 19:2626-2636. Hubbard J. Magnetism of iron. II. Physical Review B 1979; 20: 4584. Hubbard J. Magnetism of nickel. Physical Review B 1981; 23: 5974.

[61] Hasegawa H. Single-Site Functional-Integral Approach to Itinerant-Electron Ferromagnetism. J. Phys. Soc. Jpn. 1979; 46: 1504-1514. Hasegawa H. Single-Siet Spin Fluctuation Theory of Itinerant-Electron Systems with Narrow Bands. J. Phys. Soc. Jpn. 1980; 49: $178-188$.

[62] Gyorffy BL, Stocks GM. Concentration Waves and Fermi Surfaces in Random Metallic Alloys. Phys Rev Lett 1983; 50: 374-7. DOI: http://dx.doi.org/10.1103/PhysRevLett.50.374

[63] Staunton JB, Johnson DD and Pinski FJ. Compositional short-range ordering in metallic alloys: Band-filling, charge-transfer, and size effects from a first-principles all-electron Landautype theory. Phys Rev B 1994; 50:1450-72. DOI: http://dx.doi.org/10.1103/PhysRevB.50.1450

[64] Niklasson AMN, Wills JM, Katsnelson MI, Abrikosov IA, Eriksson O, Johansson B. Modeling the actinides with disordered local moments. Phys Rev B 2003; 67:235105. DOI: http://dx.doi.org/10.1103/PhysRevB.67.235105

[65] Vitos L, Korzavyi PA, Johansson B. Stainless steel optimization from quantum mechanical calculations. Nature Mater 2003; 2:25-28. doi:10.1038/nmat790

[66] Alling B, Marten T, Karimi A, Abrikosov IA. Comparison of thermodynamic properties of cubic Cr-xAlxN and Ti1-xAlxN from first-principles calculations. J Appl Phys 2007; 102: 044314-8. http://dx.doi.org/10.1063/1.2773625

[67] Lind H, Forsén R, Alling B, Ghafoor N, Tasnadi F, Johansson MP, et.al. Improving thermal stability of hard coating films via a concept of multicomponent alloying. Appl Phys Lett 2011; 99: 091903-3. DOI: 10.1063/1.3631672

[68] Anisimov VI, Zaanen J, Andersen OK. Band theory and Mott insulators: Hubbard U instead of Stoner. Phys Rev B 1991; 44: 943. DOI: http://dx.doi.org/10.1103/PhysRevB.44.943 [69] Dudarev SL, Botton GA, Savrasov SY, Humphreys CJ, and Sutton AP. Electron-energyloss spectra and the structural stability of nickel oxide: An LSDA+U study. Phys Rev B 1998; 57:1505. DOI: http://dx.doi.org/10.1103/PhysRevB.57.1505 
[70] Held K, Keller G, Eyert V, Vollhardt D, Anisimov VI. Mott-Hubbard Metal-Insulator Transition in Paramagnetic V2O3: An LDA+DMFT (QMC) Study. Phys Rev Lett 2001; 86: 5345. DOI: http://dx.doi.org/10.1103/PhysRevLett.86.5345

[71] Park H, Millis AJ, Marianetti CA. Site-Selective Mott Transition in Rare-Earth-Element Nickelates. Phys Rev Lett 2012; 109:156402.

DOI: http://dx.doi.org/10.1103/PhysRevLett.109.156402

[72] Alling B, Marten T, Abrikosov IA. Effect of magnetic disorder and strong electron correlations on the thermodynamics of CrN. Phys Rev B 2010; 82:184430.

DOI: http://dx.doi.org/10.1103/PhysRevB.82.184430

[73] Taylor DW. Vibrational Properties of Imperfect Crystals with Large Defect Concentrations. Phys Rev 1967; 156:1017-29. DOI: http://dx.doi.org/10.1103/PhysRev.156.1017

[74] Zunger A, Wei SH, Ferreira LG, Bernard JE. Special quasirandom structures. Phys Rev Lett 1990; 65:353. DOI: http://dx.doi.org/10.1103/PhysRevLett.65.353

[75] Ruban AV, Razumovskiy VI. Spin-wave method for the total energy of paramagnetic state. Phys Rev B 2012; 85:174407. DOI: http://dx.doi.org/10.1103/PhysRevB.85.174407

[76] Ponomareva AV, Gornostyrev YN, Abrikosov IA. Ab initio calculation of the solution enthalpies of substitutional and interstitial impurities in paramagnetic fcc Fe. Phys Rev B 2014; 90: 014439. DOI: http://dx.doi.org/10.1103/PhysRevB.90.014439

[77] Alling B, Hultberg L, Hultman L, Abrikosov IA. Strong electron correlations stabilize paramagnetic cubic Cr1-xAlxN solid solutions. Appl Phys Lett 2013; 102:031910-4.

DOI:10.1063/1.4788747

[78] Ruban AV, Razumovskiy VI, Körmann F. Erratum: Spin-wave method for the total energy of paramagnetic state. Phys Rev B 2014; 89:179901(E).

DOI: http://dx.doi.org/10.1103/PhysRevB.89.179901

[79] Ma PW, Woo CH, Dudarev SL. Large-scale simulation of the spin-lattice dynamics in ferromagnetic iron. Phys Rev B 2008; 78:024434.

DOI: http://dx.doi.org/10.1103/PhysRevB.78.024434

[80] Ma PW, Dudarev SL. Dynamic magnetocaloric effect in bcc iron and hcp gadolinium. Phys Rev B 2014; 90:024425. DOI: http://dx.doi.org/10.1103/PhysRevB.90.024425

[81] Perera D, Landau DP, Nicholson DM, Stocks GM, Eisenbach M, Yin J, et.al. Phononmagnon interactions in body centered cubic iron: A combined molecular and spin dynamics study. J Appl Phys 2014; 115:17D124. http://dx.doi.org/10.1063/1.4863488 
[82] Yin J, Eisenbach M, Nicholson DM, Rusanu A. Effect of lattice vibrations on magnetic phase transition in bcc iron. Phys Rev B 2012; 86:214423.

DOI: http://dx.doi.org/10.1103/PhysRevB.86.214423

[83] Shulumba N, Alling B, Hellman O, Mozafari E, Steneteg P, Oden M, Abrikosov IA. Vibrational free energy and phase stability of paramagnetic and antiferromagnetic CrN from ab initio molecular dynamics. Phys Rev B 2014; 89: 174108.

DOI: http://dx.doi.org/10.1103/PhysRevB.89.174108

[84] Hellman O, Abrikosov IA, Simak SI. Lattice dynamics of anharmonic solids from first principles. Phys Rev B 2011; 84:180301(R). Hellman O, Steneteg P, Abrikosov IA, Simak SI. Phys Rev B 2013; 87:104111.

[85] Filippetti A, Hill NA. Magnetic Stress as a Driving Force of Structural Distortions: The Case of CrN. Phys Rev Lett 2000; 85: 5166.

DOI: http://dx.doi.org/10.1103/PhysRevLett.85.5166

[86] Körmann F, Dick A, Grabowski B, Hickel T, Neugebauer J. Atomic forces at finite magnetic temperatures: Phonons in paramagnetic iron. Phys Rev B 2012; 85:125104.

DOI: http://dx.doi.org/10.1103/PhysRevB.85.125104

[87] Körmann F, Grabowski B, Dutta B, Hickel T, Mauger L, Fultz B, et.al. Temperature Dependent Magnon-Phonon Coupling in bcc Fe from Theory and Experiment. Phys Rev Lett 2014; 113:165503. DOI: http://dx.doi.org/10.1103/PhysRevLett.113.165503

[88] Chen Q and Sundman B. Modeling of thermodynamic properties for Bcc, Fcc, liquid, and amorphous iron. J Phase Equilibria 2001; 22: 631-644. DOI 10.1007/s11669-001-0027-9

[89] Belozerov AS, Leonov I, Anisimov VI. Magnetism of iron and nickel from rotationally invariant Hirsch-Fye quantum Monte Carlo calculations. Phys Rev B 2013; 87:125138. DOI: http://dx.doi.org/10.1103/PhysRevB.87.125138

[90] Zhang H, Johansson B, Vitos L. Density-functional study of paramagnetic iron. Phys Rev B 2011; 84:140411(R). DOI: http://dx.doi.org/10.1103/PhysRevB.84.140411

[91] Okatov SV, Kuznetsov AR, Gornostyrev YN, Urtsev VN, Katsnelson MI. Effect of magnetic state on the $\gamma-\alpha$ transition in iron: First-principles calculations of the Bain transformation path. Phys Rev B 2009; 79: 094111.

DOI: http://dx.doi.org/10.1103/PhysRevB.79.094111

[92] Körmann F, Dick A, Grabowski B, Hallstedt B, Hickel T, Neugebauer J. Free energy of bcc iron: Integrated ab initio derivation of vibrational, electronic, and magnetic contributions. Phys Rev B 2008; 78: 033102. DOI: http://dx.doi.org/10.1103/PhysRevB.78.033102 
[93] Glazyrin K, Pourovskii LV, Dubrovinsky L, Narygina O, McCammon C, Hewener B, Schünemann V, et.al. Importance of Correlation Effects in hcp Iron Revealed by a PressureInduced Electronic Topological Transition. Phys Rev Lett 2013; 110:117206. DOI: http://dx.doi.org/10.1103/PhysRevLett.110.117206

[94] Cort G, Taylor RD, Willis JO. Search for magnetism in hcp epsilon -Fe. J Appl Phys 1982; 53:2064-2065. Papandrew AB, Lucas MS, Stevens R, Halevy I, Fultz B, Hu MY, et.al. Absence of Magnetism in Hcp Iron-Nickel at 11 K. Phys Rev Lett 2006; 97: 087202.

[95] Pourovskii LV, Mravlje J, Ferrero M, Parcollet O, Abrikosov IA. Impact of electronic correlations on the equation of state and transport in $\varepsilon$-Fe. Phys Rev Lett, submitted, arXiv: $1312.3654 \mathrm{v} 1$.

[96] Ruban AV, Belonoshko AB, Skorodumova NV. Impact of magnetism on Fe under Earth's core conditions. Phys Rev B 2013; 87:014405.

DOI: http://dx.doi.org/10.1103/PhysRevB.87.014405

[97] Pourovskii LV, Miyake T, Simak SI, Ruban AV, Dubrovinky L, Abrikosov IA. Electronic properties and magnetism of iron at the Earth's inner core conditions. Phys Rev B 2013; 87:115130. DOI: http://dx.doi.org/10.1103/PhysRevB.87.115130

[98] Belonoshko AB, Ahuja R, Johansson B. Stability of the body-centred-cubic phase of iron in the Earth's inner core. Nature 2003; 424:1032-1034. doi:10.1038/nature01954

[99] Vočadlo L, Alfè D, Gillan MJ, Wood IG, Brodholt JP, Price GD. Possible thermal and chemical stabilization of body-centred-cubic iron in the Earth's core . Nature 2003; 424: 536539. doi:10.1038/nature01829

[100] Lin JF, Heinz DL, Campbell AJ, Devine JM, Shen G. Iron-silicon alloy in Earth's core? Science 2002; 295: 313-315. DOI: 10.1126/science.1066932

[101] Dubrovinsky L, Dubrovinskaia N, Narygina O, Kuznetzov A, Prakapenka V, Vitos L, et.al. Experimental Evidences for Body-Centred-Cubic Phase of Iron-Nickel Alloy in the Earth's Core. Science 2007; 316:1880-1883. DOI: 10.1126/science.1142105

[102] Tateno S, Hirose K, Ohishi Y, Tatsumi Y. The structure of iron in Earth's inner core. Science 2010; 330:359-361. DOI: 10.1126/science.1194662

[103] Staunton JB, Poulter J, Ginatempo B, Bruno E, Johnson DD. Incommensurate and Commensurate Antiferromagnetic Spin Fluctuations in Cr and Cr Alloys from Ab Initio Dynamical Spin Susceptibility Calculations. Phys Rev Lett 1999; 82:3340.

DOI: http://dx.doi.org/10.1103/PhysRevLett.82.3340 
[104] Torchio R, Kvashnin YO, Pascarelli S, Mathon O, Marini C, Genovese L, et al. X-Ray Magnetic Circular Dichroism Measurements in Ni up to 200 GPa: Resistant Ferromagnetism. Phys Rev Lett 2011; 107: 237202. DOI: http://dx.doi.org/10.1103/PhysRevLett.107.237202

[105] Sergueev I, Dubrovinsky L, Ekholm M, Vekilova OY, Chumakov AI, Zając M, et.al. Hyperfine Splitting and Room-Temperature Ferromagnetism of Ni at Multimegabar Pressure. Phys Rev Lett 2013; 111:157601. DOI: http://dx.doi.org/10.1103/PhysRevLett.111.157601

[106] Marco ID, Minár J, Chadov S, Katsnelson MI, Ebert H, Lichtenstein AI. Correlation effects in the total energy, the bulk modulus, and the lattice constant of a transition metal: Combined local-density approximation and dynamical mean-field theory applied to $\mathrm{Ni}$ and $\mathrm{Mn}$. Phys Rev B 2009; 79: 115111. DOI: http://dx.doi.org/10.1103/PhysRevB.79.115111

[107] Körmann F, Dick A, Hickel T, Neugebauer J. Role of spin quantization in determining the thermodynamic properties of magnetic transition metals. Phys Rev B 2011; 83:165114. DOI: http://dx.doi.org/10.1103/PhysRevB.83.165114

[108] Körmann F, Grabowski B, Söderlind P, Palumbo M, Fries SG, Hickel T, et. al. Thermodynamic modelling of chromium: strong and weak magnetic coupling. J. Phys. : Condens. Matter 2013; 25: 425401. doi:10.1088/0953-8984/25/42/425401

[109] Hickel T, Grabowski B, Körmann F, Neugebauer J. Advancing density functional theory to finite temperatures: methods and applications in steel design. J Phys.: Condens Matter 2012; 24: 053202. doi:10.1088/0953-8984/24/5/053202

[110] Marquis EA, Hyde JM, Saxey DW, Lozano-Perez S, Castro V, Hudson D, et.al. Nuclear reactor materials at the atomic scale. Materials Today 2009; 12:30-37. doi:10.1016/S1369-7021(09)70296-2

[111] Olsson P, Abrikosov IA, Vitos L, Wallenius J. Ab initio formation energies of Fe-Cr alloys. J Nucl Mater 2003; 321: 84-90. doi:10.1016/S0022-3115(03)00207-1

[112] Dench WA. Adiabatic high-temperature calorimeter for the measurement of heats of alloying. Trans Faraday Soc 1963; 59:1279-1292. DOI: 10.1039/TF9635901279

[113] Ruban AV, Korzhavyi PA, Johansson B. First-principles theory of magnetically driven anomalous ordering in bcc Fe-Cr alloys. Phys Rev B 2008; 77:094436.

DOI: http://dx.doi.org/10.1103/PhysRevB.77.094436

[114] Korzhavyi PA, Ruban AV, Odqvist J, Nilsson J-O, Johansson B. Electronic structure and effective chemical and magnetic exchange interactions in bcc Fe-Cr alloys. Phys Rev B 2009; 79: 054202. DOI: http://dx.doi.org/10.1103/PhysRevB.79.054202 
[115] Ruban AV and Razumovskiy VI. First-principles based thermodynamic model of phase equilibria in bcc Fe-Cr alloys. Phys Rev B 2012; 86:174111.

DOI: http://dx.doi.org/10.1103/PhysRevB.86.174111

[116] Gorbatov OI, Razumov IK, Gornostyrev YN, Razumovskiy VI, Korzhavyi PA, Ruban AV. Role of magnetism in Cu precipitation in $\alpha$-Fe. Phys Rev B 2013; 88:174113.

DOI: http://dx.doi.org/10.1103/PhysRevB.88.174113

[117] Sax CR, Schönfeld B, Ruban AV. Interactions and phase transformations in Fe-Pd. Phys Rev B 2014; 89:014201. DOI: http://dx.doi.org/10.1103/PhysRevB.89.014201

[118] Ponomareva AV, Ruban AV, Dubrovinskaya N, Dubrovinskiy L, Abrikosov IA. Influence of global magnetic state on chemical interactions in high-pressure hightemperature synthesis of B2-Fe2Si. Appl Phys Lett 2009; 94: 181912. DOI: 10.1063/1.3131784

[119] Lavrentiev MY, Dudarev SL, Nguyen-Manh D. Magnetic cluster expansion model for high-temperature magnetic properties of iron and iron-chromium alloys. J Appl Phys 2011; 109:07E123. http://dx.doi.org/10.1063/1.3556900

[120] Ponomareva AV, Ruban AV, Vekilova OY, Simak SI, Abrikosov IA. Effect of pressure on phase stability in Fe-Cr alloys. Phys Rev B 2011; 84: 094422.

DOI: http://dx.doi.org/10.1103/PhysRevB.84.094422

[121] Lavrentiev MY, Wrobel JS, Nguyen-Manh D, Dudarev SL. Magnetic and thermodynamic properties of face-centered cubic Fe-Ni alloys. Phys Chem Chem Phys 2014; 16:16049- 16059. DOI: 10.1039/C4CP01366B

[122] Johnson DD, Pinski FJ, Stocks GM. Effects of chemical and magnetic disorder in Fe.50Mn.50. J Appl Phys 1988; 63:3490. http://dx.doi.org/10.1063/1.340746

[123] Reyes-Huamantinco A, Puschnig P, Ambrosch-Draxl C, Peil OE, Ruban AV. Stackingfault energy and anti-Invar effect in Fe-Mn alloy from first principles. Phys Rev B 2012; 86:060201(R). DOI: http://dx.doi.org/10.1103/PhysRevB.86.060201

[124] Gebhardt T, Music D, Hallstedt B, Ekholm M, Abrikosov IA, Vitos L, et.al. Ab initio lattice stability of fcc and hcp Fe-Mn random alloys. J Phys.: Condens . Matter 2010; 22: 295402. doi: 10.1088/0953-8984/22/29/295402

[125] Ekholm M, Abrikosov IA. Structural and magnetic ground-state properties of $\gamma$-FeMn alloys from ab initio calculations. Phys Rev B 2011; 84: 104423.

DOI: http://dx.doi.org/10.1103/PhysRevB.84.104423

[126] Dick A, Körmann F, . Hickel T, Neugebauer J. Ab initio based determination of thermodynamic properties of cementite including vibronic, magnetic, and electronic excitations. Phys Rev B 2011; 84:125101. DOI: http://dx.doi.org/10.1103/PhysRevB.84.125101 
[127] Gebhardt T, Music D, Ekholm M, Abrikosov IA, Vitos L, Dick A, et.al. The influence of additions of $\mathrm{Al}$ and $\mathrm{Si}$ on the lattice stability of fcc and hcp Fe-Mn random alloys. J Phys.: Condens Matter 2011; 23:246003.

[128] Reeh S, Kasprzak M, Klusmann CD, Stalf F, Music D, Ekholm M, et. al. Elastic properties of fcc Fe-Mn-X (X = Cr, Co, Ni, Cu) alloys studied by the combinatorial thin film approach and ab initio calculations. J Phys.: Condens Matter 2013; 25: 245401 and references wherein. doi: 10.1088/0953-8984/25/24/245401

[129] Sluiter MHF. First-Principles Modelling of Phase Transformations in Steel", Ch. 23 in Part VI "Modelling of Phase Transformations. Eds. Pereloma EV and Edmonds DV. Woodhead Publishing Ltd.; 2012, 365-404.

[130] Gorbatov OI, Okatov SV, Gornostyrev YN, Korzhavyi PA, Ruban AV. Effect of magnetism on the solubility of 3d elements in BCC iron: Results of first-principle investigations. The Physics of Metals and Metallography 2013; 114: 642-653.

[131] Ding H, Razumovsky VI Asta M. Self- Diffusion Anomaly in Ferromagnetic Metals: A Density-Functional-Theory Investigation of Magnetically Ordered and Disordered Fe and Co. Acta Mater 2014; 70:130-136.

[132] Ponomareva AV, Gornostyrev YN, Abrikosov IA. Interaction energies of carbon impurities in paramagnetic $\gamma$-Fe”, ZHETP 2015; 147: in press

[133] Lobo JA, Geiger GH. Thermodynamics of carbon in austenite and Fe-Mo austenite. Metallurgical Transaction A 1976; 7:1359. Smith RP. J Amer Chem Soc 1946; 68: 1163-75. Slane JA, Wolverton C, Gibala R. Experimental and theoretical evidence for carbon-vacancy binding in austenite. Metallurgical and Materials Transactions A 2004, 35: 2239-2245. Ellis T, Davidson IM, Bodsworth C. Some Thermodynamic Properties of Carbon in Austenit. J Iron Steel Inst 1963; 210: 582-587. Bouchard D, Kirkaldy JS. Utilization of a thermogravimetric technique to determine low solubilities of gases in alloys: application to $\mathrm{Fe}-\mathrm{N}$ and $\mathrm{Ni}-\mathrm{N}$ alloys between 700 and $1000^{\circ} \mathrm{C}$. Journal of Alloys and Compounds 1999; 283:311-319. Hillert M, Jarl M. A thermodynamic analysis of the iron- nitrogen system. Met Trans 1975; 6A: 553-59.

[134] Blanter MS. Strain-induced interaction of dissolved atoms in $\gamma$-Fe. Journal of Alloys and Compounds 1999; 291:167-174. Bhadeshia HKD. Carbon-carbon interactions in iron. J. of Materials Science 2004; 39:3949-3955. Gavrilyuk VG. Raspredelenie ugleroda v stali. Kiev: Naukova Dumka; 1987. p. 208.

[135] Shorikov AO, Pchelkina ZV, Anisimov VI, Skornyakov SL, Korotin MA. Orbitalselective pressure-driven metal to insulator transition in $\mathrm{FeO}$ from dynamical mean-field theory. Phys Rev B 2010; 82:195101. DOI: http://dx.doi.org/10.1103/PhysRevB.82.195101 
[136] Ohta K, Cohen RE, Hirose K, HauleK , Shimizu K, Ohishi Y. Experimental and Theoretical Evidence for Pressure-Induced Metallization in FeO with Rocksalt-Type Structure. Phys Rev Lett 2012; 108:026403. DOI: http://dx.doi.org/10.1103/PhysRevLett.108.026403

[137] Kunes J, Korotin DM, Korotin MA, Anisimov VI, Werner P. Pressure-Driven MetalInsulator Transition in Hematite from Dynamical Mean-Field Theory. Phys Rev Lett 2009; 102:146402. DOI: http://dx.doi.org/10.1103/PhysRevLett.102.146402

[138] Gall D, Shin CS, R. Haasch RT, Petrov I, Greene JE. Band gap in epitaxial NaClstructure CrN (001) layers. J Appl Phys 2002; 91:5882-5886.

http://dx.doi.org/10.1063/1.1466528

[139] Zuev LB, Danilov VI, Barannikova SA. Pattern formation in the work hardening process of single alloyed g-Fe crystals. Int. J. Plasticity; 2001; 17: 47-63.

[140] Barannikova SA, Ponomareva AV, Zuev LB, Vekilov YK, Abrikosov IA. Significant correlation between macroscopic and microscopic parameters for the description of localized plastic flow auto-waves in deforming alloys. Solid State Commun 2012; 152:784-787.

doi:10.1016/j.ssc.2012.01.044

[141] Zhang H, Wang G, Punkkinen MP, Hertzman S, Johansson B, Vitos L. Elastic anomalies in Fe-Cr alloys. J Phys.: Condens. Matter 2013; 25:195501. doi: 10.1088/09538984/25/19/195501 (2013).

[142] Narsu B, Wang G, Johansson B, Vitos L. Large magneto-chemical-elastic coupling in highly magnetostrictive Fe-Ga alloys. Appl Phys Lett 2013; 103:231903.

http://dx.doi.org/10.1063/1.4838657

[143] Wang G, Hu Q-M, Kokko K, Johansson B, Vitos L. Ab initio investigation of the elastic properties of Ni3Fe. Phys Rev B 2013; 88:174205. DOI:

http://dx.doi.org/10.1103/PhysRevB.88.174205

[144] Sandström R, Korzhavyi P. Use of elastic constants based on ab initio computation in materials optimisation of austenitic stainless steels. Canadian Metallurgical Quarterly 2014; 53:282-291, and references wherein.

DOI: http://dx.doi.org/10.1179/1879139514Y.0000000138. 